\title{
PROBING THE PHYSICS OF NARROW LINE REGIONS IN ACTIVE GALAXIES. II. THE SIDING SPRING SOUTHERN SEYFERT SPECTROSCOPIC SNAPSHOT SURVEY (S7)
}

\author{
Michael A. Dopita ${ }^{1,2}$, Prajval Shastri ${ }^{3}$, Rebecca Davies ${ }^{1}$, Lisa Kewley ${ }^{1,4}$, Elise Hampton ${ }^{1}$, Julia Scharwächter ${ }^{5}$, \\ Ralph Sutherland $^{1}$, Preeti Kharb ${ }^{3}$, Jessy Jose ${ }^{3}$, Harish Bhatt ${ }^{3}$, S. Ramya ${ }^{3}$, Chichuan Jin ${ }^{6}$, Julie Banfield ${ }^{7}$, \\ Ingyin Zaw ${ }^{8}$, Stéphanie Juneau ${ }^{9}$, Bethan James ${ }^{10}$, and Shweta SRivastava ${ }^{11}$ \\ ${ }^{1}$ RSAA, Australian National University, Cotter Road, Weston Creek, ACT 2611, Australia; Michael.Dopita@anu.edu.au \\ ${ }^{2}$ Astronomy Department, King Abdulaziz University, P.O. Box 80203, Jeddah, Saudi Arabia \\ ${ }^{3}$ Indian Institute of Astrophysics, Koramangala 2 B Block, Bangalore 560034, India \\ ${ }^{4}$ Institute for Astronomy, University of Hawaii, 2680 Woodlawn Drive, Honolulu, HI, USA \\ ${ }^{5}$ LERMA, Observatoire de Paris, CNRS, UMR 8112, 61 Avenue de l'Observatoire, F-75014 Paris, France \\ ${ }^{6}$ Qian Xuesen Laboratory for Space Technology, Beijing, China \\ ${ }^{7}$ CSIRO Astronomy \& Space Science, P.O. Box 76, Epping NSW, 1710 Australia \\ ${ }^{8}$ New York University (Abu Dhabi), 70 Washington Square South, New York, NY 10012, USA \\ ${ }^{9}$ CEA-Saclay, DSM/IRFU/SAp, F-91191 Gif-sur-Yvette, France \\ ${ }^{10}$ Institute of Astronomy, Cambridge University, Madingley Road, Cambridge CB3 OHA, UK \\ ${ }^{11}$ Astronomy and Astrophysics Division, Physical Research Laboratory, Ahmedabad 380009, India \\ Received 2014 November 2; accepted 2015 January 7; published 2015 March 24
}

\begin{abstract}
Here we describe the Siding Spring Southern Seyfert Spectroscopic Snapshot Survey (S7) and present results on 64 galaxies drawn from the first data release. The S7 uses the Wide Field Spectrograph mounted on the ANU $2.3 \mathrm{~m}$ telescope located at the Siding Spring Observatory to deliver an integral field of $38 \times 25$ arcsec at a spectral resolution of $R=7000$ in the red $(530-710 \mathrm{~nm})$, and $R=3000$ in the blue $(340-560 \mathrm{~nm})$. From these data cubes we have extracted the narrow-line region spectra from a 4 arcsec aperture centered on the nucleus. We also determine the $\mathrm{H} \beta$ and $\left[\mathrm{O}_{\mathrm{III}}\right] \lambda 5007$ fluxes in the narrow lines, the nuclear reddening, the reddening-corrected relative intensities of the observed emission lines, and the $\mathrm{H} \beta$ and $[\mathrm{O}$ III $] \lambda 5007$ luminosities determined from spectra for which the stellar continuum has been removed. We present a set of images of the galaxies in [O III $] \lambda 5007,[\mathrm{~N} \mathrm{II}] \lambda 6584$, and $\mathrm{H} \alpha$, which serve to delineate the spatial extent of the extended narrow-line region and also to reveal the structure and morphology of the surrounding $\mathrm{H}$ il regions. Finally, we provide a preliminary discussion of those Seyfert 1 and Seyfert 2 galaxies that display coronal emission lines in order to explore the origin of these lines.
\end{abstract}

Key words: galaxies: abundances - galaxies: active - galaxies: ISM - galaxies: jets - galaxies: Seyfert

Supporting material: machine-readable table

\section{INTRODUCTION}

It has been understood for many years that massive black holes are ubiquitous in the centers of the more massive disk and elliptical galaxies, and that there exists an intimate connection between the black hole mass and the host galaxy measured either through the bulge mass, luminosity, or velocity dispersion (Magorrian et al. 1998; Ferrarese \& Merritt 2000; Gebhardt et al. 2000; Tremaine et al. 2002; McConnell \& Ma 2013). Furthermore, it has been established that these relationships were already in place by $z \sim 2$ (Bennert et al. 2011), demonstrating that the build up of mass in the host galaxy bulges was contemporaneous with the growth of their central black holes.

Our understanding of the more recent mass feeding of these nuclear black holes remains somewhat sketchy. We can measure the luminosity of the active galactic nucleus (AGN) in the local universe, but this provides the product of the black hole mass and the Eddington fraction. In terms of the electron scattering opacity, the Eddington fraction is defined as $f_{\text {Edd. }}=L_{\mathrm{BH}} /\left(1.25 \times 10^{38} \mathrm{erg} \mathrm{s}^{-1}\left[M_{\mathrm{BH}} / M_{\odot}\right]\right)$. In practice, it is very difficult to separate the two variables of black hole mass and Eddington fraction. In addition, the issues of orientation and obscuration sometimes render it difficult to even estimate the luminosity of the central engine, unless a very long baseline spectral energy distribution (SED) is available. According to the "standard" unified model of AGNs (Antonucci \& Barvainis 1990; Antonucci 1993) and its extensions-which attempt to account for the effect of the Eddington fraction of the accretion rate (Dopita 1997)-the Seyfert 1 galaxies are seen pole-on relative to the accretion disk, and these display very broad permitted lines originating in rapidly moving gas close to the central engine. In the Seyfert 2 galaxies, the thick accretion disk obscures the central engine, and an extended narrow-line region (ENLR), often confined within an "ionization cone," is observed. In this geometry, the ENLR can be readily observed even when the central engine is very heavily obscured at optical wavelengths (Kewley et al. 2001). The fundamental problem with the original unified model with a thick accretion disk is that, if continuous, such a disk could not support itself against collapse in the vertical direction. Now the clumpy torus model (Nenkova et al. 2002; Elitzur 2006; Ramos Almeida et al. 2009) is more generally accepted, but this more physically motivated model still operates to obscure the central engine over a range of angles and also to confine the escape of the EUV radiation to the polar directions.

The properties of the ENLR can provide vital clues about the nature of the central black hole, and the mechanisms that produce the EUV continuum. Seyfert galaxies are known to occupy a very restricted range of line ratios when plotted on the 
well-known BPT diagram (Baldwin et al. 1981), which plots $[\mathrm{N} \mathrm{II}] \lambda 6584 / \mathrm{H} \alpha$ vs. [O III] $\lambda 5007 / \mathrm{H} \beta$ or alternatively, using the other diagrams introduced by Veilleux \& Osterbrock (1987) involving either the $\left[\mathrm{S} \mathrm{II}^{\mathrm{II}}\right] \lambda 6717,31 / \mathrm{H} \alpha$ ratio or the $\left[\mathrm{O}_{\mathrm{I}}\right] \lambda 6300 /$ $\mathrm{H} \alpha$ ratio in place of the $[\mathrm{N} \mathrm{II}] \lambda 6584 / \mathrm{H} \alpha$ ratio. It now seems that this is because the ENLR is, in general, radiation pressure dominated (Dopita et al. 2002a; Groves et al. 2004a, 2004b). In this model, radiation pressure (acting upon both the gas and the dust) compresses the gas close to the ionization front so that at high enough radiation pressure, the density close to the ionization front scales as the radiation pressure, and the local ionization parameter $(U$, the ratio of the density of ionizing photons to the ion density) in the optically emitting ENLR becomes constant. This results in an optical ENLR spectrum, which is virtually independent of the input ionization parameter. For dusty ENLRs, the radiation pressure comes to dominate the gas pressure for $\log U \gtrsim-2.5$, and the emission spectrum in the optical becomes invariant with the input ionization parameter for $\log U \gtrsim-0.5$. In this condition, the observed density in the ENLR should drop off in radius in lockstep with the local intensity of the radiation field; $n_{e} \propto r^{-2}$, and the EUV luminosity can be inferred directly using density and the radial distance.

In the first paper of this series, Dopita et al. (2014) applied these ideas to a test-case example Seyfert, NGC 5427, to determine how well the EUV spectrum, luminosity, and black hole mass can be determined from an analysis of the narrowline spectrum of the nucleus and its associated ENLR. In this paper they utilized an idea originally put forward by Evans \& Dopita (1987) to use an analysis of the $\mathrm{H}$ II regions surrounding the AGN to constrain the chemical abundance of the ENLR. By eliminating chemical abundance as a free variable, the gross features of the EUV spectrum (between 13.6 and $\sim 150 \mathrm{eV}$ ) can be inferred using a method similar to the energy balance or Stoy technique that has long been used to estimate the effective temperature of stars in planetary nebulae (Stoy 1933; Kaler 1976; Preite-Martinez \& Pottasch 1983). This relies on the fact that as the radiation field becomes harder, the heating per photoionization increases, and the sum of the fluxes of the forbidden lines becomes greater relative to the recombination lines. Furthermore, additional constraints are available because individual line ratios are sensitive in different ways to the form of the EUV spectrum. These properties can be exploited to infer the form of the EUV spectrum, and the application of this technique will be the subject of future papers in this series.

Apart from the emission-line spectrum, the dynamical structure of the ENLR is also of great interest. Most ENLR show velocity dispersions of up to a few hundred $\mathrm{km} \mathrm{s}^{-1}$. It is not yet clear what fraction of this velocity dispersion is due to outflows originally powered by circumnuclear starbursts, what is the contribution to the velocity dispersion of radiatively driven outflows (Cecil et al. 2002; Dopita et al. 2002a; Mullaney et al. 2009), or what fraction is generated by the cocoon shocks powered by the overpressure of relativistic plasma derived from the radio jets (Bicknell et al. 1998; Tadhunter et al. 2014).

At a sufficiently great distance, the spectra of the ENLR and the $\mathrm{H}$ II regions become mixed together within a single resolution element. This mixing has been investigated by Scharwächter et al. (2011) and Dopita et al. (2014), and further quantified by Davies et al. (2014a, 2014b). From the BPT diagram (Baldwin et al. 1981) and the Veilleux \& Osterbrock
(1987) diagnostics, it is possible to both clearly define the zone of influence of the AGN and to quantify the total luminosity of the ENLR. A future paper will investigate this mixing of the Seyferts with ENLR presented here.

The other major class of AGN in the local universe is the low-ionization nuclear emission-line regions (LINERs), first defined as a class by Heckman (1980). The host galaxies of these objects are generally large ellipticals, although they are also found in some nearby spirals such as M 81. We now understand that LINERs represent AGNs with low Eddington fractions (Kewley et al. 2006), but we are still a long way from understanding why this should produce the characteristic LINER spectrum in the optical. The most likely mechanisms is shocks (Koski \& Osterbrock 1976; Fosbury et al. 1978; Baldwin et al. 1981) or photoionization by a power-law or thermal Bremstrahhlung continuum with a low-ionization parameter (Ferland \& Netzer 1983).

Low-level LINER emission has now been detected in a large fraction of elliptical galaxies (Phillips et al. 1986; Veron-Cetty \& Veron 1986; Ho 1996; Ho et al. 1997). Surveys by O'Connell \& Dressel (1978) and Heckman (1980) confirmed a strong correlation between bright LINER emission and powerful compact nuclear radio sources. In most of these bright LINERs, virtually all radio emission comes from flatspectrum, compact self-absorbed synchrotron sources (Condon \& Dressel 1978).

It has recently become clear that the frequently detected "extended" LINER emission is not necessarily of the same origin as the true nuclear LINER activity. Maoz et al. (1998) found that the LINER class is not homogeneous in its UV properties - some objects exhibit strong emission lines, while others display a UV spectrum consistent with an old stellar population. Indeed, for extended LINER emission, Yan \& Blanton (2012) proved that the extended emission is inconsistent with ionization from a central object, and that most likely post-AGB stars are required to provide the ionization. This idea was originally proposed by Binette et al. (1994), and was recently developed with CALIFA integral field spectroscopic data by Singh et al. (2013). Such low ionization extended regions perhaps should be more appropriately referred to as "LIERs"!

From the above discussion it should be clear that a great deal of interesting physics can be derived from a survey of the ENLR of nearby Seyferts and of LINER galaxies, provided that the survey has adequate spectral resolution to investigate the dynamics, adequate spectral coverage to provide complete strong-line diagnostics and to allow unambiguous photoionization modeling, and adequate spatial resolution to both resolve the spatial and dynamical structure of the ENLR and to isolate individual $\mathrm{H}$ in region complexes. This clearly defines a survey that can only be undertaken using an Integral Field Unit (IFU).

Until now, Seyfert and LINER galaxies have mainly been identified through single-aperture spectroscopic surveys, of which the most notable is the SDSS survey. In its seventh data release DR7 (Abazajian et al. 2009), the SDSS spectroscopic catalog covers more than $10^{4} \mathrm{deg}^{2}$ of the high-latitude sky and contains many thousands of Seyferts and LINERS, as well as $\sim 10^{5}$ quasars. The physical properties of the Seyferts and LINERs in this catalog were recently been effectively examined by Zhang et al. (2013). 
Surveys probing the extent of the ENLR of Seyfert galaxies are rather sparse. Most of these are derived from images taken in the $[\mathrm{O} \mathrm{III}] \lambda 5007$ emission line-which is very strong in the ENLR - and often using $\mathrm{H} \alpha+\left[\mathrm{N}_{\text {II }}\right] \lambda 6584$ images. This allows one to distinguish the $\mathrm{H}$ II regions from the ENLR (Haniff et al. 1988; Wilson et al. 1988; Pogge 1989; Evans et al. 1996; Davies et al. 2014b, 2014a). The most extensive ground-based surveys of both the extent and morphology of Seyferts are still those of Mulchaey et al. (1996a, 1996b). Falcke et al. (1998) used the WFPC2 imager on the Hubble Space Telescope (HST) to identify extended [O III] $\lambda 5007$ emission in seven Seyfert 2 galaxies, as did Schmitt et al. (2003a, 2003b) in a sample of 60 Seyfert galaxies (22 Sy 1 and 38 Sy 2 galaxies), selected based on their far-infrared properties. Finally, we should mention the important UV survey of Muñoz Marín et al. (2007), which probed the central regions of 75 Seyfert galaxies imaged in the near-UV with the Advanced Camera for Surveys (ACS) of the $H S T$ at an average resolution of $\sim 10 \mathrm{pc}$.

Here, we present initial results from the first data release of the Siding Spring Southern Seyfert Spectroscopic Snapshot Survey (S7). This comprises an integral field survey of more than 130 galaxies in total, of which 64 galaxies are included in this first data release. In this paper we concentrate on a presentation of the narrow-band images of the Seyfert galaxies in our sample, and identify the objects showing pronounced ENLR and/or circumnuclear star-formation activity. We also present the nuclear spectra of all objects extracted from a 4 arcsec diameter aperture, and present the measured reddening-corrected emission-line fluxes between [O II] $\lambda \lambda 3737,9$ and $[\mathrm{S}$ II $] \lambda \lambda 6717,31$. In Section 2 we describe the characteristics of this survey, in Section 2.2 the observational data set, and in Section 3 the reduction techniques used. Our results are given in Section 4. In Section 4.1 we present the results of fitting stellar continua and a three-component emission-line model to our spectra. This allows the extraction of the nuclear properties. In Section 4.2 we examine the systematics of Seyfert 1 and Seyfert 2 galaxies with coronal-line emission. The results from the narrow-band images extracted from the data cubes are presented in Section 4.3, in which we note the angular and physical sizes of the ENLR, and probe the relationship between the ENLR and the $\mathrm{H}$ in regions. The results on individual galaxies are given in Section 5. Finally in Section 6 we present our conclusions. In this paper, we assume $H_{0}=71 \mathrm{~km} \mathrm{~s}^{-1} \mathrm{Mpc}^{-1}$, following the $7 \mathrm{yr} W M A P$ results (Larson et al. 2011).

\section{AN INTEGRAL FIELD AGN SURVEY}

\subsection{The S7 Survey}

The S7 survey is an integral field survey in the optical of $\sim 140$ southern Seyfert and LINER galaxies. It uses the Wide Field Spectrograph (WiFeS) mounted on the Nasmyth focus of the ANU $2.3 \mathrm{~m}$ telescope. This instrument is described in Dopita et al. (2007) and its performance is discussed in Dopita et al. (2010). WiFeS provides data cubes across a field of $38 \times 25$ arcsec at a spatial resolution of 1.0 arcsec. It covers the waveband $340-710 \mathrm{~nm}$ with the unusually high resolution of $R=7000$ in the red $(530-710 \mathrm{~nm})$ and $R=3000$ in the blue $(340-570 \mathrm{~nm})$. The typical throughput of the instrument (top of the atmosphere to back of the detector) is 20-35\% (Dopita et al. 2010), which provides an excellent sensitivity to faint low surface brightness ENLR features, while the high resolution $\left(\sim 50 \mathrm{~km} \mathrm{~s}^{-1}\right)$ in the red enables the different velocity components of emission lines to be clearly separated.

The S7 survey is by no means a complete survey of southern Seyfert, LINERs, and other active galaxies, rather it offers a reasonably representative sample of radio-detected AGNs in the nearby universe. The objects for the S7 were selected from the Véron-Cetty \& Véron (2006) catalog of active galaxies, which remains the most comprehensive compilation of active galaxies in the literature. In the future we wish to investigate the interaction of the bipolar plasma jets with the NLR and the ISM of the host galaxy, so we limited the sample to galaxies with radio flux densities high enough to permit radio aperture synthesis observations. We adopted the following selection criteria.

1. Decl. $<10^{\circ}$ to avoid making observations at too great a zenith distance. At a zenith distance of $60^{\circ}$, the atmospheric dispersion between 3500 and $7000 \AA$ is nearly 6 arcsec, which would seriously compromise the effective field coverage.

2. Galactic latitude $>|20|$ degrees (with a few exceptions in the case of objects known to have an associated ENLR). This galactic latitude requirement is set to avoid excessive galactic extinction.

3. Radio flux density at $20 \mathrm{~cm} \gtrsim 20 \mathrm{mJy}$ for those targets with decl. N of $-40 \mathrm{deg}$, which have NVSS measurements.

4. Redshift $<0.02$. This $(D<80 \mathrm{Mpc})$ ensures that the spatial resolution of the data is better than $400 \mathrm{pc} \mathrm{arcsec}^{-1}$, which is sufficient to resolve the ENLR, and to ensure that the important diagnostic $\left[\mathrm{S}_{\mathrm{II}}\right]$ lines are still within the spectral range of the WiFeS high-resolution red grating.

\subsection{The Observations}

Each target was observed with the nucleus centered in the WiFeS aperture, at a position angle either close to the major axis of the galaxy, or along the axis of the radio jet and/or the ENLR, where this axis was known. The observing strategy was as follows. Galaxies were observed in pairs that were separated on the sky by no more than about $15^{\circ}$. In each galaxy we selected a nearby blank sky reference region that could then be used for the purpose of sky subtraction in either galaxy.

A typical complete series of exposures comprising a single observing sequence would be $1 \times$ Sky region\#1, $3 \times$ Galaxy\#1, $1 \times$ Sky region\#1, Bias, Copper-Argon Arc Calibration, $1 \times$ Sky region\#2, $3 \times$ Galaxy\#2, and $1 \times$ Sky region\#2. The exposure times of the individual WiFeS frames ranged from $800 \mathrm{~s}$ to $1000 \mathrm{~s}$, depending on the observing conditions and the length of the night. Observing in this manner allowed us to combine the first two sky frames with the third sky frame to provide the sky reference for Galaxy \#1, while the second sky frame was combined with the third and the fourth to provide the sky reference for Galaxy \#2, giving an on-target observation efficiency of close to $60 \%$.

The absolute photometric calibration was made using the STIS spectrophotometric standard stars. ${ }^{12}$ In addition, a number of B-type telluric standards were observed to correct for the $\mathrm{OH}$ and $\mathrm{H}_{2} \mathrm{O}$ telluric absorption features in the red. The separation of these features by molecular species allowed for a more accurate telluric correction, which accounted for night to

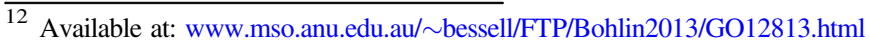


Table 1

Log of the Observations

\begin{tabular}{|c|c|c|c|c|c|c|c|c|c|}
\hline $\begin{array}{l}\text { Object } \\
\text { Name }\end{array}$ & $\begin{array}{c}\text { R.A. } \\
(\text { J2000) }\end{array}$ & $\begin{array}{r}\text { Decl. } \\
(\mathrm{J} 2000)\end{array}$ & $\bar{z}$ & Type & $\begin{array}{l}\text { Type } \\
\text { Veron }\end{array}$ & $\mathrm{PA}^{\circ}$ & $\begin{array}{c}\text { Date of } \\
\text { Observation }\end{array}$ & $\begin{array}{l}\text { Exposure } \\
\text { Time }(\mathrm{s})\end{array}$ & $\begin{array}{l}\text { Seeing } \\
(\operatorname{arcsec})\end{array}$ \\
\hline PKS 0056-572 & 005846.7 & -565909.9 & 1.46 & QSO & S1 & 0 & 2013 Nov 1 & 3000 & 1.1 \\
\hline NGC 424 & 011127.5 & -380500.7 & 0.0118 & Sey 2, Cor! $!^{\mathrm{a}}, \mathrm{B}(\mathrm{H} \alpha)^{\mathrm{b}}$ & $\mathrm{S} 1 \mathrm{~h}$ & 90 & 2013 Aug 12 & 3000 & 1.8 \\
\hline NGC 613 & 013418.1 & -292503.0 & 0.0049 & Sey 2 & $\mathrm{~S} ?$ & 90 & 2013 Nov 3 & 3000 & 2.2 \\
\hline IC 1657 & 011407.0 & -323903.0 & 0.0119 & Sey 2 & $\mathrm{~S} 2$ & 0 & 2013 Aug 8 & 3000 & 2.1 \\
\hline MARK 573 & 014357.8 & 022103.0 & 0.0172 & Sey 2, Cor. $^{\mathrm{c}}, \mathrm{B}(\mathrm{H} \alpha)$ & S1h & 90 & 2013 Nov 4 & 3000 & 2.0 \\
\hline IRAS 01475-0740 & 015002.6 & -072550.0 & 0.0177 & Sey 2 & $\mathrm{~S} 1 \mathrm{~h}$ & 90 & 2013 Aug 9 & 3000 & 1.8 \\
\hline NGC 833 & 020920.8 & -100759.3 & 0.0129 & LINER & $\mathrm{S} 2$ & 90 & 2013 Aug 4 & 3000 & 3.0 \\
\hline NGC 835 & 020924.6 & -100808.3 & 0.0129 & LINER & S2 & 0 & 2013 Aug 3 & 3000 & 1.6 \\
\hline IC 1816 & 023151.1 & -364011.9 & 0.0169 & Sey 2 & $\mathrm{~S} 2$ & 90 & 2013 Nov 4 & 3000 & 2.7 \\
\hline NGC 1052 & 024104.9 & $-08 \quad 1521.0$ & 0.0050 & LINER & $\mathrm{S} 3 \mathrm{~b}$ & 90 & 2013 Nov 2 & 3000 & 1.5 \\
\hline NGC 1097 & 024619.2 & -301619.0 & 0.0042 & LINER & $\mathrm{S} 3 \mathrm{~b}$ & 0 & 2013 Nov 4 & 3000 & 2.7 \\
\hline NGC 1125 & 025140.3 & -163904.0 & 0.0109 & Sey 2 & $\mathrm{~S} 2$ & 90 & 2013 Nov 1 & 3000 & 1.2 \\
\hline NGC 1204 & 030440.1 & -122022.9 & 0.0154 & $\mathrm{SB}^{\mathrm{d}}$ & $\mathrm{S} 2$ & 60 & 2013 Nov 3 & 3000 & 2.3 \\
\hline NGC 1566 & 042001.0 & -545616.7 & 0.0050 & Sey $1, \mathrm{~B}(\mathrm{H} \alpha)$ & $\mathrm{S} 1.5$ & 90 & 2013 Nov 1 & 3000 & 1.3 \\
\hline ESO 202-G23 & 042800.0 & -475424.0 & 0.0165 & LINER or Sy 2 & $\mathrm{~S} 3$ & 0 & 2013 Nov 3 & 3000 & 2.5 \\
\hline NGC 1808 & 050742.1 & -373033.8 & 0.0033 & SB & $\mathrm{H} 2$ & 0 & 2013 Nov 2 & 3000 & 1.1 \\
\hline MARK 1210 & 080405.9 & 050649.3 & 0.0135 & Sey 2, Cor., B $(\mathrm{H} \alpha)$ & S2 & 90 & 2014 Mar 2 & 2700 & 1.6 \\
\hline NGC 2617 & 083538.8 & -040518.0 & 0.0142 & Sey 1 & $\mathrm{~S} 1.8$ & 90 & 2014 Mar 2 & 2700 & 1.6 \\
\hline MCG -01-24-012 & 092046.3 & -080322.0 & 0.0196 & Sey 2 & S2 & 45 & 2014 Apr 5 & 3000 & 1.7 \\
\hline MCG -05-23-004 & 093107.4 & -302130.3 & 0.0086 & LINER & S3 & 90 & 2014 Apr 5 & 3000 & 1.2 \\
\hline MCG -05-23-008 & 094413.4 & -285055.0 & 0.0084 & LINER & $\mathrm{S}$ & 90 & 2014 Apr 8 & 1800 & 1.7 \\
\hline NGC 2992 & 094542.0 & -141933.4 & 0.0077 & Sey $2, \mathrm{~B}(\mathrm{H} \alpha)$ & S1.9 & 45 & 2014 Apr 5 & 3000 & 2.0 \\
\hline MARK 1239 & 095219.1 & -013642.8 & 0.0199 & Sey 1,Cor!, B $(\mathrm{H} \alpha)$ & S1n & 0 & 2014 Mar 2 & 2700 & 1.8 \\
\hline NGC 3100 & 100040.9 & -313952.0 & 0.0088 & LINER & $\mathrm{S} 3$ & 0 & 2014 Apr 5 & 2700 & 2.5 \\
\hline IC 2560 & 101618.7 & -333350.0 & 0.0097 & Sey 2, Cor., B $(\mathrm{H} \alpha)$ & S2 & 45 & 2014 Apr 8 & 1800 & 1.5 \\
\hline MCG -06-23-038 & 102945.6 & -382055.0 & 0.0152 & Sey 2 & $\mathrm{~S}$ & 90 & 2014 Mar 2 & 2700 & 2.3 \\
\hline IRAS $11215-2806$ & 112409.8 & -282349.0 & 0.0140 & Sey 2 & S2 & 135 & 2014 Apr 7 & 2700 & 2.0 \\
\hline NGC 3783 & 113902.2 & $-3744 \quad 17.0$ & 0.0090 & Sey 1, Cor! & S1.5 & 0 & 2014 Apr 7 & 1800 & 2.5 \\
\hline NGC 4303 & 122155.4 & 042831.0 & 0.0052 & SB or LINER & $\mathrm{S} 2$ & 0 & 2014 & 1800 & 1.3 \\
\hline NGC 4404 & 122616.2 & -074051.0 & 0.0186 & LINER & S3 & 90 & 2014 Apr 5 & 3000 & 1.8 \\
\hline 3 C 278 & 125436.9 & -123328.6 & 0.0150 & Elliptical & S3 & 90 & 2014 Apr 5 & 3000 & 2.1 \\
\hline NGC 5253 & 133956.0 & -313813.0 & 0.0014 & SB & $\mathrm{H} 2$ & 0 & 2013 Aug 5 & $3000^{\mathrm{e}}$ & 1.9 \\
\hline NGC 5506 & 141314.8 & $\begin{array}{lll}-03 & 12 & 27.0\end{array}$ & 0.0062 & Sey 2, Cor & S1i & 90 & 2013 Aug 6 & 3000 & 0.9 \\
\hline NGC 5597 & 142428.0 & -164544.0 & 0.0089 & $\mathrm{SB}+\mathrm{WR}^{\mathrm{f}}$ & $\mathrm{H} 2$ & 45 & 2014 Apr 8 & 1800 & 1.1 \\
\hline NGC 5664 & 143343.7 & $-1437 \quad 10.5$ & 0.0152 & $\mathrm{SB}+$ Sey 2 & $\mathrm{~S} 2$ & 30 & 2014 Apr 5 & 3000 & 2.0 \\
\hline NGC 5728 & 144224.7 & $-17 \quad 1507.0$ & 0.0093 & Sey 2,Cor & S1.9 & 120 & 2014 Apr 8 & 1800 & 1.2 \\
\hline NGC 5757 & 144646.4 & -190442.7 & 0.0089 & SB & $\mathrm{H} 2$ & 0 & 2013 Aug 3 & 3000 & 2.0 \\
\hline NGC 6000 & 154949.6 & -292313.0 & 0.0073 & SB & $\mathrm{H} 2$ & 0 & 2014 Apr 5 & 3000 & 1.8 \\
\hline ESO 137-G34 & 163513.9 & -580448.3 & 0.0091 & Sey 2 & $\mathrm{~S} 2$ & 90 & 2013 Aug 10 & 3000 & 1.4 \\
\hline ESO 138-G01 & 165120.1 & -591348.0 & 0.0091 & Sey 2, Cor.,B $(\mathrm{H} \alpha)$ & S2 & 90 & 2014 Apr 8 & 1800 & 1.1 \\
\hline NGC 6221 & 165246.0 & -591301.0 & 0.0050 & $\mathrm{SB}+$ Sey 2 & $\mathrm{~S} 2$ & 90 & 2014 Apr 8 & 1800 & 1.2 \\
\hline NGC 6300 & 171659.3 & -624914.2 & 0.0037 & Sey 2 & S2 & 90 & 2013 Aug 10 & 3000 & 1.4 \\
\hline FAIRALL 49 & 183658.2 & -592408.0 & 0.0200 & Sey $2, \mathrm{~B}(\mathrm{H} \alpha)$ & S1h & 90 & 2014 Apr 8 & 1800 & 1.2 \\
\hline ESO 103-G35 & 183820.4 & -652538.4 & 0.0130 & Sey 2 & $\mathrm{~S} 2$ & 90 & 2013 Aug 9 & 3000 & 1.5 \\
\hline FAIRALL 51 & 184453.8 & -622151.0 & 0.0142 & Sey 1, Cor! & $\mathrm{S} 1.5$ & 0 & 2013 Aug 6 & 3000 & 1.3 \\
\hline NGC 6812 & 194524.8 & -552050.2 & 0.0154 & LINER & $\mathrm{S} ?$ & 90 & 2013 Aug 4 & 3000 & 1.6 \\
\hline ESO 339-G11 & 195737.5 & -375608.0 & 0.0192 & Sey 2, Cor., B $(\mathrm{H} \alpha)$ & S2 & 90 & 2013 Aug 5 & 3000 & 1.5 \\
\hline NGC 6860 & 200847.0 & -610601.0 & 0.0149 & Sey 1 & $\mathrm{~S} 1.5$ & 0 & 2013 Aug 12 & 3000 & 2.6 \\
\hline NGC 6890 & 201818.1 & -444824.2 & 0.0081 & Sey 2 , Cor. & S1.9 & 0 & 2013 Aug 10 & 3000 & 1.1 \\
\hline NGC 6915 & 202746.1 & -030437.6 & 0.0189 & LINER & $\mathrm{S} 3$ & 90 & 2013 Aug 5 & 3000 & 1.5 \\
\hline NGC 6926 & 203306.2 & -020137.8 & 0.0196 & Sey 2 & S2 & 0 & 2013 Aug 9 & 3000 & 1.1 \\
\hline IC5063 & 205202.2 & -570407.5 & 0.0113 & Sey $2, \mathrm{~B}(\mathrm{H} \alpha)$ & S1h & 90 & 2013 Aug 12 & 3000 & 2.0 \\
\hline IC 1368 & 211412.6 & 021040.7 & 0.0130 & Sey 2 & $\mathrm{~S} 2$ & 90 & 2013 Aug 6 & 3000 & 1.5 \\
\hline NGC 7130/IC 5135 & 214819.4 & -345703.3 & 0.0161 & $\mathrm{SB}+\operatorname{Sey} 2$ & S1.9 & 90 & 2013 Aug 3 & 3000 & 1.3 \\
\hline NGC 7213 & 220916.2 & $\begin{array}{lll}-47 & 10 & 00.7\end{array}$ & 0.0058 & LINER & $\mathrm{S} 3 \mathrm{~b}$ & 90 & 2013 Aug 5 & 3000 & 1.3 \\
\hline IC 1459 & 225710.5 & -362745.0 & 0.0060 & LINER & $\mathrm{S} 3$ & 0 & 2013 Aug 3 & 3000 & 1.7 \\
\hline NGC 7469 & 230316.0 & 085224.5 & 0.0163 & Sey 1 , Cor. & S1.5 & 90 & 2013 Aug 10 & 3000 & 1.3 \\
\hline NGC 7496 & 230947.3 & -432540.5 & 0.0055 & $\mathrm{SB}+\operatorname{Sey} 2$ & $\mathrm{~S} 2$ & 0 & 2013 Nov 1 & 3000 & 1.2 \\
\hline NGC 7552 & 231610.8 & -423505.0 & 0.0054 & SB & $\mathrm{H} 2$ & 90 & 2013 Aug 6 & 3000 & 1.5 \\
\hline NGC 7591 & $2318 \quad 16.3$ & 063510.0 & 0.0165 & LINER & $\mathrm{S}$ & 0 & 2013 Aug 5 & 3000 & 1.1 \\
\hline NGC 7582 & $2318 \quad 23.4$ & $-42 \quad 22 \quad 13.6$ & 0.0053 & Sey $2+$ SB & S1i & 0 & 2013 Nov 2 & 3000 & 1.3 \\
\hline NGC 7590 & $23 \quad 1854.5$ & $-42 \quad 1407.8$ & 0.0052 & Sey 2 & $\mathrm{~S} 2$ & 0 & 2013 Nov 3 & 3000 & 1.5 \\
\hline
\end{tabular}


Table 1

(Continued)

\begin{tabular}{|c|c|c|c|c|c|c|c|c|c|}
\hline $\begin{array}{l}\text { Object } \\
\text { Name }\end{array}$ & $\begin{array}{l}\text { R.A. } \\
(\mathrm{J} 2000)\end{array}$ & $\begin{array}{r}\text { Decl. } \\
(\mathrm{J} 2000)\end{array}$ & $z$ & Type & $\begin{array}{l}\text { Type } \\
\text { Veron }\end{array}$ & $\mathrm{PA}^{\circ}$ & $\begin{array}{c}\text { Date of } \\
\text { Observation }\end{array}$ & $\begin{array}{l}\text { Exposure } \\
\text { Time (s) }\end{array}$ & $\begin{array}{l}\text { Seeing } \\
(\operatorname{arcsec})\end{array}$ \\
\hline NGC 7679 & 232846.8 & 033045.0 & 0.0171 & $\mathrm{SB}+$ Sey 2 & S1.9 & 90 & 2013 Nov 4 & 3000 & 1.8 \\
\hline NGC 7714 & 233614.2 & 020917.6 & 0.0093 & SB & $\mathrm{H} 2$ & 0 & 2013 Aug 5 & 3000 & 1.5 \\
\hline
\end{tabular}

${ }^{\text {a }}$ Strong and high-excitation coronal lines present.

${ }^{\mathrm{b}}$ Broad component detected in $\mathrm{H} \alpha$.

c Coronal lines present.

${ }^{\mathrm{d}} \mathrm{SB}=$ Starburst

e Bright lines saturated.

${ }^{\mathrm{f}} \mathrm{WR}=$ Wolf Rayet features present.

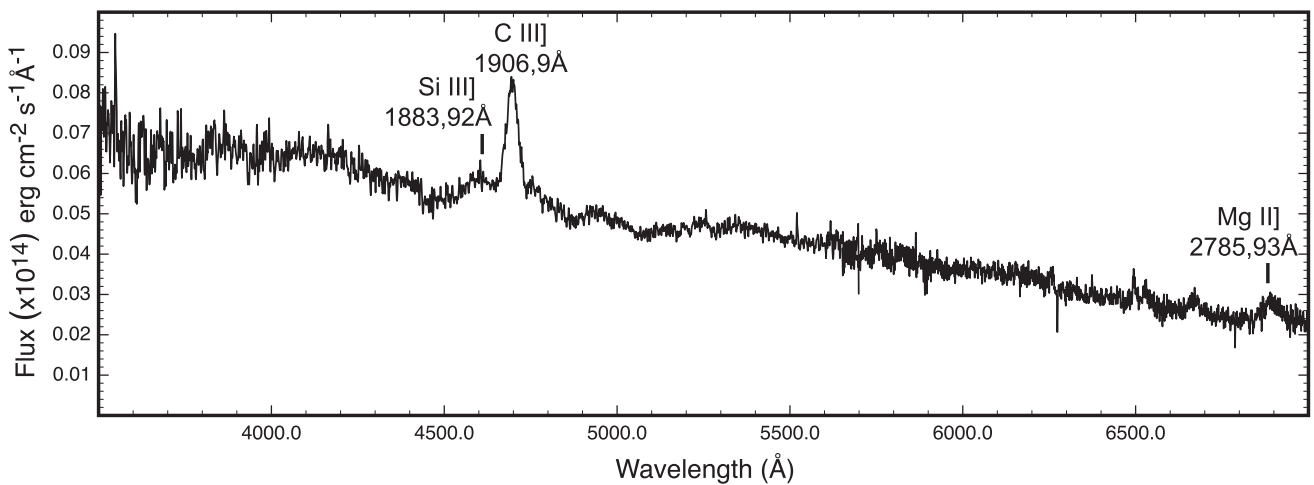

Figure 1. Measured spectrum and line identifications for PKS 0056-572. We conclude that this object may be a QSO at a redshift of $z=1.46$. However, see the Note Added in Proof

night variations in the column density of these two species. In addition, a set of calibration flat fields and twilight sky flats were taken in each of the five observing runs in which the data was collected.

In Table 1 we present the observing log, which lists the date of observation, the total exposure time in all three subexposures, and the mean seeing during each observation as measured in the auto guider. This table also includes the classification of the nuclear activity, and a comparison with the type given in Véron-Cetty \& Véron (2006). A number of mismatches are evident. Most spectacular is the case of PKS 0056-572, which was classified as a Seyfert 1, but is actually a QSO at a redshift of $z=1.46$ (see Figure 1). In addition, a number of LINER galaxies are classified in the Véron-Cetty \& Véron (2006) catalog as S 2. One galaxy, 3C278, has no detectable emission features, and appears to be an Elliptical galaxy on the basis of its stellar continuum. In Table 1 we have also noted the cases where coronal emission features are evident. The [Fe VII] 6087, $5721 \AA$ lines are visible in all of these, but in the objects with strong high-excitation coronal features, the [Fe XIV] $5303 \AA$ and [Fe X] $6374 \AA$ lines are also prominent. Table 1 provides information on the observed extent of the ENLR, and notes the cases among the Seyfert 2 galaxies in which the fitting procedure (see Section 3.2, below) indicated the presence of an underlying broad component to the $\mathrm{H} \alpha$ profile, which might be the signature of a highly extinguished or hidden broad-line region (BLR).

\section{DATA REDUCTION}

The data were reduced using the standard PyWiFeS pipeline written for the instrument and fully described in Childress et al. (2014). In brief, this produces a summed data cube for each object wavelength calibrated and evenly sampled in wavelength, sensitivity corrected in both the spatial and spectral directions (including telluric corrections), photometrically calibrated using standard stars, and from which cosmic ray events have been removed. The red and blue arm data were reduced independently, producing spectral data cubes regularly sampled at $0.77 \AA$ in the blue and $0.44 \AA$ in the red.

In this paper we restrict ourselves to an extraction of emission-line images from the whole data cube and an examination of the nuclear spectra. However, future papers will examine the issues of mixing between the ENLR and $\mathrm{H}$ II regions within a single spaxel, the ENLR structure and dynamics, the chemical abundances derived from the $\mathrm{H}$ II regions, and the constraints that can be placed on the nature of the central engine from the spectroscopy.

\subsection{Images}

We have extracted continuum-subtracted emission-line images from the data cubes using QFitsView V3.1 rev. $741 .{ }^{13}$ Our objective was to separate the emission-line objects in the field by their excitation mechanism. We therefore combined the $\left[\mathrm{O}_{\text {III }}\right] \lambda 5007$ image (blue channel) with the $[\mathrm{N}$ II] $\lambda 6584$ image (green channel), and placed the $\mathrm{H} \alpha$ image in the red channel. With this scheme, the high metallicity $\mathrm{H}$ II regions found in the vicinity of Seyfert nuclei appear red, gold, or sometimes yellow, because their [O III] $\lambda 5007$ emission is generally weak and the $\left[\mathrm{N}_{\mathrm{II}}\right] \lambda 6584$ line is usually weaker than $\mathrm{H} \alpha$. The Seyfert nuclei, on the other hand, appear blue,

\footnotetext{
13 QFitsView v3.1 is a FITS file viewer using the QT widget library and was developed at the Max Planck Institute for Extraterrestrial Physics by Thomas Ott.
} 
turquoise, or sometimes green because their [O III] $\lambda 5007$ emission is very strong, and in many cases the $[\mathrm{N}$ II] $\lambda 6584$ emission is as strong or even stronger than $\mathrm{H} \alpha$.

\subsection{Circum-nuclear Spectra}

From the data cube we have extracted the circumnuclear spectra of each galaxy from a fixed 4 arcsec diameter aperture using QFitsView. This aperture-typically of order $1 \mathrm{kpc}$ at the galaxy, was chosen to provide the integrated nuclear fluxes of all galaxies, regardless of seeing conditions. Any remaining residual of the night sky lines of $\left[\mathrm{O}_{\mathrm{I}}\right] \lambda 5577.3,\left[\mathrm{O}_{\mathrm{I}}\right] \lambda 6300.3$ and $\left[\mathrm{O}_{\mathrm{I}}\right] \lambda 6363.8$ were removed by hand. In addition-in the case of the small fraction of data obtained in non-photometric conditions - a scaling factor was sometimes applied to the red spectrum using the relative scaling determined in the spectral overlap region between 5500 and $5600 \AA$. The data were smoothed by a boxcar function with a 1:3:5:3:1 weighting function to remove noise within a given resolution element, and then shifted to the rest wavelength using the measured wavelength of the $[\mathrm{N}$ II $] \lambda 6584$ line.

\section{RESULTS}

\subsection{Circum-nuclear Spectra}

We used the IFS toolkit LZIFU (I.-T. Ho et al. 2014, in preparation) to derive gas and stellar kinematics from the nuclear spectra. LZIFU uses the penalized pixel-fitting routine (PPXF Cappellari \& Emsellem 2004) to perform simple stellar population (SSP) synthesis fitting to model the continuum, and fits the emission lines as Gaussians using the LevenbergMarquardt least-squares technique (Markwardt 2009). We employ the theoretical SSP libraries from González Delgado et al. (2005) assuming the Padova isochrones. For the gas velocity and velocity dispersion, we simultaneously fit up to three Gaussians to each of the optical emission lines, and constrain each of the components to have the same velocity and velocity dispersion for every emission line. Seyfert 1 galaxies were the exception; the broad component was fit to only the Balmer and Helium recombination lines. We fix the ratios [O III] $\lambda \lambda 4959 / 5007$ and $\left[\mathrm{N}_{\mathrm{II}}\right] \lambda \lambda 6548 / 6584$ to their theoretical values given by quantum mechanics (Dopita \& Sutherland 2003).

The choice of the number of Gaussians to fit is driven by the quality of the fit. First one, then two, and, if needed, three Gaussian profiles are fit to the narrow lines. If there is no improvement in the residuals by the addition of another component, the number of Gaussians is fixed at the lower number. In the case of the Seyfert 1 galaxies, either one broad component or a broad plus intermediate-width component are required to fit the broad-line profile, which restricts the quality of the fit that can be obtained in the NLR. In general, the coronal emission in those objects that display these lines are well fit by the intermediate component.

The reddening is determined independently for the stellar continuum component and the emission lines. Since the measured emission-line fluxes determined for the higher members of the Balmer series may be in error due to the competition between the emission in the line and the absorption in the underlying stellar continuum (which is very sensitive to the age of the younger stellar components), we avoided using the higher members of the Balmer series in the extinction determination, and used only the $\mathrm{H} \alpha / \mathrm{H} \beta$ ratio to determine $A_{V}$ using the formulation given in Vogt et al. (2013). We assumed the $\mathrm{H} \alpha / \mathrm{H} \beta$ ratio to be its theoretical value at a temperature of $10,000 \mathrm{~K}$ and a density of $10^{3} \mathrm{~cm}^{-3}$, which is 2.86 (Dopita \& Sutherland 2003).

In Figure 2 we show the quality of the fits achieved in typical spectra representing each of the four major classes of object covered by S7: a Seyfert 1, a Seyfert 2, a LINER galaxy, and a Starburst galaxy. This figure shows how both young and old stellar populations can be well fit. In addition, double-line profiles can be reproduced, as shown by ESO 137-G34 in Figure 3. For Seyfert 1 broad lines, the broad lines are not particularly well fit, but these are only used to allow a measurement of the NLR spectrum in these objects. The residuals on the fit are typically only of order of $1-2 \%$, except in the case of the $\mathrm{NaD}$ absorption lines, which are not modelled.

In order to determine the flux and extinction in the narrowline region, and to extract the narrow-line spectrum, we only used the two narrowest components of the three-component fit. The justification for this is that the broad component was used only in the case of the recombination lines of the Seyfert 1 BLR (see, for example, Figure 2, NGC 3783). These arise from the nuclear BLR, rather than in the NLR, the spectrum and emission-line ratios of which we seek to extract.

In Table 2 we give the luminosity distance, extinction, size of the effective aperture at the distance of the object, the observed $\mathrm{H} \beta$ and $\left[\mathrm{O}_{\mathrm{III}}\right] \lambda 5007$ fluxes, the inferred nuclear luminosities in these two emission lines, and the approximate size of the ENLR based on the region of the data cube in which the $[\mathrm{O}$ III $] \lambda 5007$ line is detected with a signal to noise greater than 5. In Table 3, we list the velocity widths (FWHM) of the three fitted components, and in Table 4 we give the measured emission-line fluxes of our objects, sorted according to their R.A.

\subsection{Seyferts with Coronal Lines}

In our sample, and as noted in Table 1, we find a number of objects of both the Seyfert 1 and Seyfert 2 types that have coronal emission from species such as [Fe V], [Fe VII], [Fe X], and $[\mathrm{Fe} \mathrm{XIV}]$ in their spectra. In Figure 4 we show a number of these, representative of the Seyfert 2 objects that display coronal emission. It is evident from the figure that the relative strength and excitation of the coronal emission is correlated with the $\mathrm{H} \alpha$ line width, and with the electron density in the [O III]-emitting region, as evidenced by the [O III $] \lambda \lambda 4363 / 5007$ ratio. The density in the $\left[\mathrm{O}\right.$ III] zone must exceed $10^{6} \mathrm{~cm}^{-3}$ in the densest example (MARK 1239), while the low-excitation gas as revealed by the $\left[\mathrm{S}_{\mathrm{II}}\right] \lambda \lambda 6717 / 6731$ ratio remains around $n_{e} \sim 10^{4} \mathrm{~cm}^{-3}$, and the strength of the $[\mathrm{S} \mathrm{II}]$ lines and the [N II] $\lambda 6584$ line relative to the broad component of $\mathrm{H} \alpha$ also changes systematically with $\mathrm{H} \alpha$ line width. These lowexcitation lines clearly arise in a region that is physically distinct from the region that emits the coronal species.

A confirmation that the Seyferts with coronal lines form a distinct population is obtained by an examination of the Baldwin et al. (1981; BPT) and Veilleux \& Osterbrock (1987) diagnostic plots. In Figure 5 we plot the objects with coronal lines (red circles) and the S7 Seyfert $2 \mathrm{~s}$ (black circles) against the AGNs from SDSS as isolated by Vogt et al. (2014). From this figure, it is evident that the objects with coronal lines form a sequence that is somewhat separated from those without. Whereas normal Seyfert $2 \mathrm{~s}$ are clustered in the upper part of the Seyfert branch as defined by the SDSS galaxies, most objects with coronal lines lie above and to the left. In general 

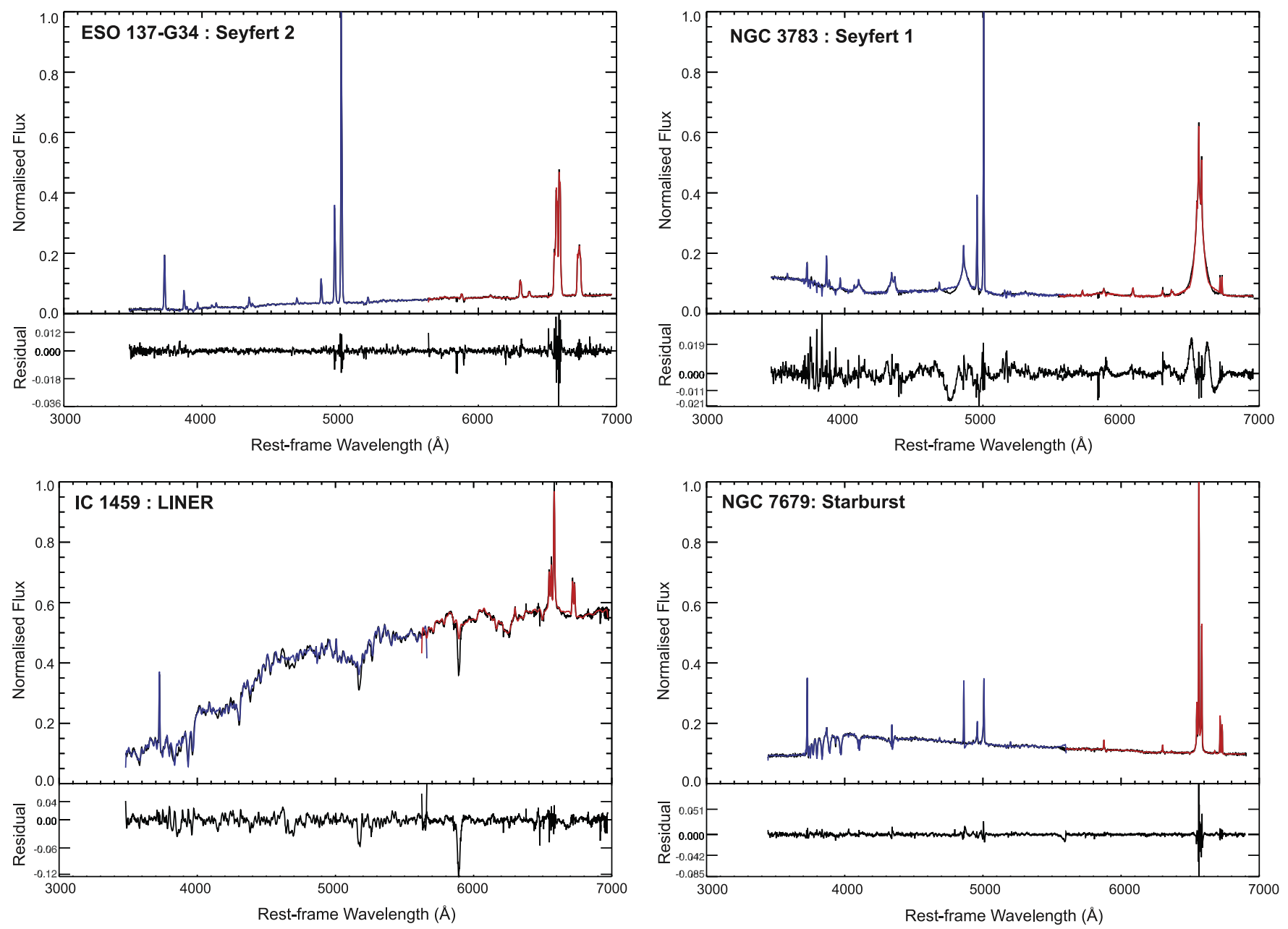

Figure 2. Representative fits to the nuclear spectra from S7. The instrumental resolution is comparable with the line thickness in these panels. We show one example of each of the major classes of object in the survey. Top left, ESO 137-G34, a typical Seyfert 2. Top right, NGC 3783, a typical Seyfert 1. The broad lines are not used when extracting the narrow-line spectrum from the fit. Bottom left, IC 1459 a typical LINER. The spectrum of the old stellar population is particularly prominent, as is the interstellar absorption in the Na D lines (not fitted). Bottom right, NGC 7679, a typical starburst or post-starbust (given the prominence of the underlying A-type stellar absorption). In this nuclear spectrum, the $[\mathrm{O}$ III] $\lambda \lambda 4959,5007$ lines are broader than the others, and we infer the presence of a very weak Seyfert 2 mixed with the $\mathrm{H}$ II region spectrum.

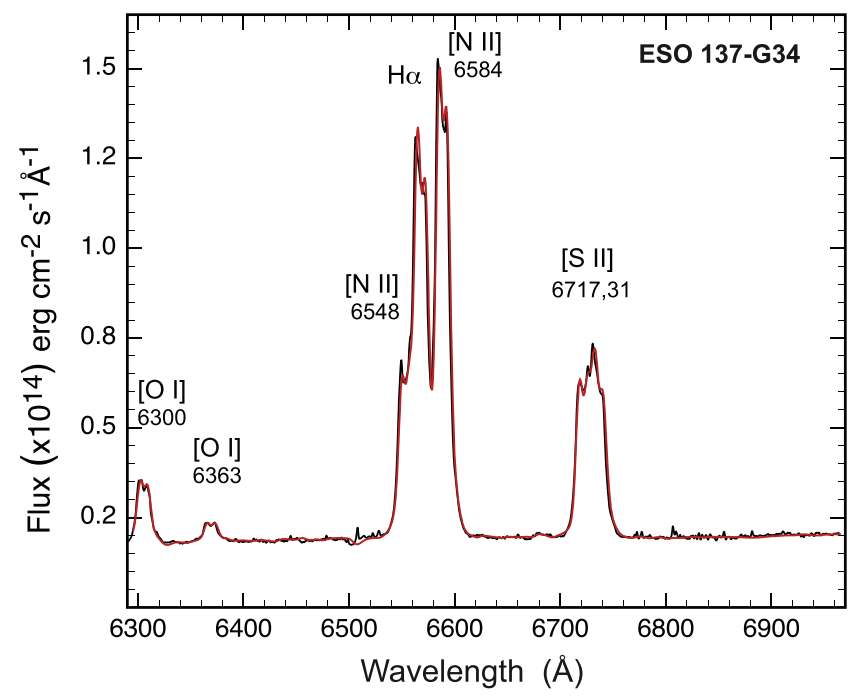

Figure 3. Fit (red line) to the observation (black line) for the Seyfert 2 ESO 137-G34 in the region of $\mathrm{H} \alpha$. This demonstrates how the double-peak nature of the emission lines in this object is very well fit by the LzIFu code. they have lower $\left[\mathrm{S}_{\mathrm{II}}\right] / \mathrm{H} \alpha$ ratios, and the more extreme objects also have lower $[\mathrm{N} \mathrm{II}] / \mathrm{H} \alpha$ and $[\mathrm{O} \mathrm{III]}] / \mathrm{H} \alpha$ ratios. This is most easily understood as an effect of density, because the forbidden line ratios appear to decrease in the order of their critical density. For example, $\left[\mathrm{S}_{\mathrm{II}}\right] / \mathrm{H} \alpha$ decreases before $[\mathrm{O}$ III $] / \mathrm{H} \beta$, which would go some way toward explaining the distribution the coronal Seyferts on Figure 4. From an inspection of the line widths, it is also clear that these are ordered according to their critical density, with $\left[\mathrm{S}_{\mathrm{II}}\right] \lambda 6717,31$ and $[\mathrm{N}$ II] $\lambda 6584$ being the narrowest, [O $\mathrm{III}] \lambda 5007$ and $\left[\mathrm{O}_{\mathrm{I}}\right] \lambda 6300$ being broader, the coronal lines broader still, and $\mathrm{H} \alpha$ the widest. However, from these observations it is not possible to disentangle the effects of rotation and outflow in determining the width of the various emission lines.

These properties are consistent with the model advocated by Mullaney et al. (2009). In this, the coronal lines arise in a dense gas that is launched from the dusty inner torus $\left(10^{17}<R / \mathrm{cm}<10^{18}\right)$ at very high local ionization parameter $\log U \sim-0.4$, and which is accelerated by radiation pressure to a terminal velocity of a few hundred $\mathrm{km} \mathrm{s}^{-1}$. At this ionization parameter the gas is Compton heated to $\sim 10^{6} \mathrm{~K}$ or 
Table 2

Derived Nuclear Extinctions, $\mathrm{H} \beta$ and $[\mathrm{O}$ III] Fluxes, Luminosities, and Extent of the Narrow-line Region

\begin{tabular}{|c|c|c|c|c|c|c|c|c|c|}
\hline Object & Type & $\begin{array}{l}\mathrm{D}_{\text {Lum. }} \\
(\mathrm{Mpc})\end{array}$ & $\begin{array}{c}A_{V} \\
\text { (mag.) }\end{array}$ & $\begin{array}{l}\text { Aperture } \\
(\mathrm{kpc})\end{array}$ & 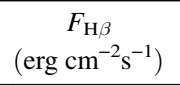 & $F_{\mathrm{O} \text { II }}$ & $\begin{array}{l}\log L_{\mathrm{H} \beta} \\
\left(\mathrm{erg} \mathrm{s}^{-1}\right)\end{array}$ & $\log L_{\mathrm{O} \text { шा }}$ & $\begin{array}{c}\text { Size ENLR } \\
(\operatorname{arcsec})\end{array}$ \\
\hline NGC 424 & Seyfert 2 & 45.6 & 1.76 & 0.87 & $5.12 \mathrm{E}-14$ & $3.19 \mathrm{E}-13$ & 40.87 & 41.67 & $18 \times 14$ \\
\hline NGC 613 & Seyfert 2 & 17.1 & 2.73 & 0.32 & $3.44 \mathrm{E}-14$ & $1.78 \mathrm{E}-14$ & 40.27 & 39.99 & $20 \times 14$ \\
\hline IC 1657 & Seyfert 2 & 46.2 & 1.65 & 0.88 & $3.56 \mathrm{E}-15$ & $6.40 \mathrm{E}-15$ & 39.68 & 39.93 & $11 \times 10$ \\
\hline MARK 573 & Seyfert 2 & 67.4 & 0.74 & 1.28 & 4.49E-14 & $4.60 \mathrm{E}-13$ & 40.71 & 41.72 & $>25 \times 15$ \\
\hline IRAS 01475-074 & Seyfert 2 & 69.7 & 2.93 & 1.32 & $9.72 \mathrm{E}-15$ & $4.86 \mathrm{E}-14$ & 41.03 & 41.73 & $10 \times 7$ \\
\hline NGC 833 & LINER & 50.0 & 0.00 & 0.95 & $5.95 \mathrm{E}-15$ & $7.26 \mathrm{E}-15$ & 39.25 & 39.33 & $\cdots$ \\
\hline NGC 835 & Seyfert 2 & 52.9 & 1.82 & 1.01 & $1.22 \mathrm{E}-14$ & $5.56 \mathrm{E}-15$ & 40.40 & 40.06 & $\cdots$ \\
\hline IC 1816 & Seyfert 2 & 68.4 & 0.72 & 1.30 & $1.56 \mathrm{E}-14$ & $1.56 \mathrm{E}-13$ & 40.25 & 41.25 & $15 \times 13$ \\
\hline NGC 1052 & LINER & 17.8 & 0.04 & 0.34 & $5.95 \mathrm{E}-14$ & $1.07 \mathrm{E}-14$ & 39.37 & 38.62 & $\cdots$ \\
\hline NGC 1097 & LINER & 15.2 & 0.45 & 0.29 & $1.05 \mathrm{E}-14$ & $8.17 \mathrm{E}-15$ & 38.66 & 38.55 & $\cdots$ \\
\hline NGC 1125 & Seyfert 2 & 42.6 & 2.46 & 0.81 & $2.00 \mathrm{E}-14$ & $1.23 \mathrm{E}-13$ & 40.71 & 41.50 & $\sim 30 \times 10$ \\
\hline NGC 1204 & SB & 61.4 & 4.64 & 1.17 & $1.99 \mathrm{E}-15$ & $7.38 \mathrm{E}-16$ & 40.98 & 40.55 & $\ldots$ \\
\hline NGC 1566 & Seyfert 1 & 20.5 & 0.09 & 0.39 & $6.47 \mathrm{E}-14$ & $1.89 \mathrm{E}-13$ & 39.55 & 40.01 & $15 \times 10$ \\
\hline ESO 202-G23 & Seyfert 2 & 68.5 & 2.56 & 1.30 & $1.52 \mathrm{E}-15$ & $2.74 \mathrm{E}-15$ & 40.05 & 40.30 & $\cdots$ \\
\hline NGC 1808 & SB & 14.0 & 3.38 & 0.27 & $4.34 \mathrm{E}-14$ & $1.11 \mathrm{E}-14$ & 40.48 & 39.89 & $\cdots$ \\
\hline MARK 1210 & Seyfert 2 & 59.5 & 1.03 & 1.13 & $5.98 \mathrm{E}-14$ & $5.74 \mathrm{E}-13$ & 40.85 & 41.83 & $<5 \times 5$ \\
\hline NGC 2617 & Seyfert 1 & 61.2 & 2.55 & 1.16 & $1.84 \mathrm{E}-14$ & $2.89 \mathrm{E}-14$ & 41.03 & 41.23 & Knot at 15 \\
\hline MCG -01-24-012 & Seyfert 2 & 86.6 & $\cdots$ & 1.65 & $\ldots$ & $\ldots$ & $\cdots$ & $\cdots$ & $9 \times 6$ \\
\hline MCG -05-23-004 & LINER & 39.9 & 0.00 & 0.76 & $4.38 \mathrm{E}-15$ & $5.45 \mathrm{E}-15$ & 38.92 & 39.01 & $\cdots$ \\
\hline MCG -05-23-008 & LINER & 39.3 & 0.00 & 0.75 & $4.56 \mathrm{E}-15$ & $3.70 \mathrm{E}-15$ & 38.92 & 38.83 & $\cdots$ \\
\hline NGC 2992 & Seyfert 2 & 36.6 & 3.78 & 0.70 & $1.55 \mathrm{E}-14$ & $8.15 \mathrm{E}-14$ & 41.05 & 41.77 & $>35 \times 25$ \\
\hline MARK 1239 & Seyfert 1 & 88.0 & 2.58 & 1.67 & $1.39 \mathrm{E}-13$ & $2.39 \mathrm{E}-13$ & 42.23 & 42.47 & $17 \times 15$ \\
\hline NGC 3100 & LINER & 40.9 & 0.00 & 0.78 & $9.43 \mathrm{E}-15$ & $8.47 \mathrm{E}-15$ & 39.27 & 39.23 & $\cdots$ \\
\hline IC 2560 & Seyfert 2 & 44.9 & 1.33 & 0.85 & $2.30 \mathrm{E}-14$ & $2.46 \mathrm{E}-13$ & 40.32 & 41.35 & $13 \times 18$ \\
\hline MCG -06-23-038 & Seyfert 2 & 67.3 & 2.47 & 1.28 & $5.50 \mathrm{E}-15$ & $3.93 \mathrm{E}-14$ & 40.55 & 41.41 & $11 \times 9$ \\
\hline IRAS 11215-2806 & Seyfert 2 & 62.9 & 1.58 & 1.20 & 4.05E-15 & $3.19 \mathrm{E}-14$ & 39.97 & 40.87 & $\sim 7 \times 7$ \\
\hline NGC 3783 & Seyfert 1 & 44.7 & 0.36 & 0.85 & 4.03E-14 & $3.82 \mathrm{E}-13$ & 40.14 & 41.12 & $\sim 18 \times 18$ \\
\hline NGC 4303 & SB or LINER & 26.3 & 1.33 & 0.50 & $3.59 \mathrm{E}-14$ & $3.31 \mathrm{E}-14$ & 40.05 & 40.02 & $\cdots$ \\
\hline NGC 4404 & LINER & 82.6 & 0.00 & 1.57 & $4.65 \mathrm{E}-15$ & $3.25 \mathrm{E}-15$ & 39.58 & 39.42 & $\cdots$ \\
\hline NGC 5506 & Seyfert 2 & 29.1 & 2.07 & 0.55 & $4.85 \mathrm{E}-14$ & $3.37 \mathrm{E}-13$ & 40.60 & 41.44 & $15 \times 12$ \\
\hline NGC 5597 & $\mathrm{SB}+\mathrm{WR}$ & 40.5 & 1.44 & 0.77 & $7.75 \mathrm{E}-14$ & $2.48 \mathrm{E}-14$ & 40.81 & 40.31 & 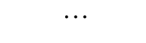 \\
\hline NGC 5664 & SB + Seyfert 2 & 66.3 & 1.94 & 1.26 & $7.68 \mathrm{E}-15$ & $2.27 \mathrm{E}-14$ & 40.45 & 40.92 & $>25 \times 25$ \\
\hline NGC 5728 & Seyfert 2 & 41.9 & 1.23 & 0.80 & $3.48 \mathrm{E}-14$ & $3.31 \mathrm{E}-13$ & 40.40 & 41.38 & Jets, $22 \times 7$ \\
\hline NGC 5757 & SB & 40.0 & 2.14 & 0.76 & $3.38 \mathrm{E}-14$ & $4.97 \mathrm{E}-15$ & 40.75 & 39.91 & $\cdots$ \\
\hline NGC 6000 & SB & 32.1 & 3.14 & 0.61 & $3.70 \mathrm{E}-14$ & $6.62 \mathrm{E}-15$ & 41.03 & 40.28 & $\cdots$ \\
\hline ESO 137-G34 & Seyfert 2 & 38.8 & 2.16 & 0.74 & $3.12 \mathrm{E}-14$ & $2.90 \mathrm{E}-13$ & 40.69 & 41.66 & $>40 \times 25$ \\
\hline ESO 138-G01 & Seyfert 2 & 38.6 & 2.12 & 0.73 & $6.98 \mathrm{E}-14$ & $5.75 \mathrm{E}-13$ & 41.02 & 41.93 & Jets $>35$ \\
\hline NGC 6221 & $\mathrm{SB}+\mathrm{Sey}$ & 21.4 & 3.49 & 0.41 & 8.29E-14 & $4.25 \mathrm{E}-14$ & 41.18 & 40.89 & $<5 \times 5$ \\
\hline NGC 6300 & Seyfert 2 & 15.7 & 4.33 & 0.30 & $2.90 \mathrm{E}-15$ & $4.29 \mathrm{E}-14$ & 39.82 & 40.99 & $\sim 7 \times 5$ \\
\hline FAIRALL 49 & Seyfert 2 & 83.1 & 3.31 & 1.58 & $5.53 \mathrm{E}-14$ & $2.09 \mathrm{E}-13$ & 42.11 & 42.68 & $28 \times 10$ \\
\hline ESO 103-G35 & Seyfert 2 & 77.7 & 2.36 & 1.48 & $6.18 \mathrm{E}-15$ & $4.55 \mathrm{E}-14$ & 40.68 & 41.54 & $\sim 26 \times 8$ \\
\hline FAIRALL 51 & Seyfert 1 & 58.5 & 0.98 & 1.11 & $3.30 \mathrm{e}-14$ & 6.09 e- 14 & 40.56 & 40.83 & $10 \times 7$ \\
\hline NGC 6812 & LINER & 62.9 & 0.05 & 1.20 & $6.00 \mathrm{E}-15$ & $9.11 \mathrm{E}-15$ & 39.47 & 39.66 & $\cdots$ \\
\hline ESO 339-G11 & Seyfert 2 & 77.7 & 3.86 & 1.48 & $6.35 \mathrm{E}-15$ & $4.99 \mathrm{E}-14$ & 41.35 & 42.24 & $\sim 9 \times 5$ \\
\hline NGC 6860 & Seyfert 1 & 60.7 & 1.04 & 1.15 & $1.02 \mathrm{E}-14$ & $5.38 \mathrm{E}-14$ & 40.11 & 40.83 & $15 \times 15$ \\
\hline NGC 6890 & Seyfert 2 & 31.2 & 1.50 & 0.59 & $1.30 \mathrm{E}-14$ & $1.42 \mathrm{E}-13$ & 39.83 & 40.87 & $\sim 8 \times 8$ \\
\hline NGC 6915 & LINER & 74.9 & 0.00 & 1.42 & $2.88 \mathrm{E}-15$ & $3.25 \mathrm{E}-15$ & 39.28 & 39.34 & 7 times 5 \\
\hline NGC 6926 & Seyfert 2 & 82.6 & 3.92 & 1.57 & $8.98 \mathrm{E}-16$ & $3.94 \mathrm{E}-15$ & 40.58 & 41.22 & $<3 \times 3$ \\
\hline IC5063 & Seyfert 2 & 45.3 & 2.28 & 0.86 & $3.07 \mathrm{E}-14$ & $2.41 \mathrm{E}-13$ & 40.87 & 41.77 & $>35 \times 25$ \\
\hline IC 1368 & Seyfert 2 & 49.6 & 5.07 & 0.94 & $1.79 \mathrm{E}-15$ & $6.46 \mathrm{E}-15$ & 40.94 & 41.49 & $<5 \times 5$ \\
\hline NGC 7130 & $\mathrm{SB}+$ Seyfert & 63.6 & 1.79 & 1.21 & $3.35 \mathrm{E}-14$ & $1.55 \mathrm{E}-13$ & 40.99 & 41.66 & $9 \times 9$ \\
\hline NGC 7213 & LINER & 21.2 & 0.17 & 0.40 & $6.81 \mathrm{E}-14$ & $8.17 \mathrm{E}-14$ & 39.64 & 39.72 & $9 \times 9$ \\
\hline IC 1459 & LINER & 21.1 & 0.00 & 0.40 & $3.66 \mathrm{E}-14$ & $2.34 \mathrm{E}-14$ & 39.29 & 39.09 & $\cdots$ \\
\hline NGC 7469 & Seyfert 1 & 62.7 & 3.47 & 1.19 & 7.44E-14 & $3.88 \mathrm{E}-13$ & 42.06 & 42.77 & $16 \times 8$ \\
\hline NGC 7496 & $\mathrm{SB}+\mathrm{Sey}$ & 19.4 & 1.45 & 0.37 & 7.07E-14 & $3.89 \mathrm{E}-14$ & 40.14 & 39.88 & $<6 \times 6$ \\
\hline NGC 7591 & LINER & 63.6 & 3.85 & 1.21 & $1.38 \mathrm{E}-15$ & $9.98 \mathrm{E}-16$ & 40.51 & 40.37 & $\cdots$ \\
\hline NGC 7582 & Seyfert $2+$ SB & 18.3 & 3.11 & 0.35 & 5.33E-14 & $2.93 \mathrm{E}-14$ & 40.69 & 40.43 & $\cdots$ \\
\hline NGC 7590 & Seyfert 2 & 18.3 & 1.89 & 0.35 & $6.03 \mathrm{E}-15$ & $2.09 \mathrm{E}-14$ & 39.21 & 39.75 & $<3 \times 3$ \\
\hline NGC 7679 & SB + Seyfert & 66.2 & 1.03 & 1.26 & $6.13 \mathrm{E}-14$ & $6.44 \mathrm{E}-14$ & 40.95 & 40.98 & $\sim 20 \times 5$ \\
\hline NGC 7714 & $\mathrm{SB}$ & 33.5 & 1.49 & 0.64 & $2.88 \mathrm{E}-13$ & $4.01 \mathrm{E}-13$ & 41.24 & 41.38 & $\cdots$ \\
\hline
\end{tabular}


Table 3

Velocity Widths $\left(\mathrm{km} \mathrm{s}^{-1}\right)$ of the Three Fitted Components to the Emission Lines

\begin{tabular}{|c|c|c|c|c|c|c|c|}
\hline$\underline{\text { Object }}$ & $V_{1}$ & $V_{2}$ & $V_{3}$ & Object & $V_{1}$ & $V_{2}$ & $V_{3}$ \\
\hline NGC 424 & 182 & 385 & $\ldots$ & NGC 5664 & 113 & $\ldots$ & $\ldots$ \\
\hline NGC 613 & 70 & 198 & $\ldots$ & NGC 5728 & 113 & $\ldots$ & $\ldots$ \\
\hline IC 1657 & 38 & 124 & $\cdots$ & NGC 5757 & 64 & 153 & $\cdots$ \\
\hline MARK 573 & 97 & 237 & $\cdots$ & NGC 6000 & 120 & 241 & $\cdots$ \\
\hline $\begin{array}{l}\text { IRAS } \\
01475\end{array}$ & 62 & 207 & $\cdots$ & ESO 127-G34 & 131 & 137 & 392 \\
\hline NGC 833 & 236 & $\cdots$ & $\cdots$ & ESO 138-G01 & 88 & 211 & $\cdots$ \\
\hline IC1816 & 122 & 127 & $\ldots$ & NGC 6221 & 51 & 210 & $\ldots$ \\
\hline NGC 1052 & 158 & 429 & $\cdots$ & NGC 6300 & 93 & 220 & $\cdots$ \\
\hline NGC 1097 & 184 & $\cdots$ & $\cdots$ & FAIRALL 49 & 77 & 150 & 494 \\
\hline NGC 1125 & 114 & 344 & $\cdots$ & ESO 103-G35 & 124 & 167 & 459 \\
\hline NGC 1204 & 41 & 123 & $\cdots$ & FAIRALL 51 & 106 & 705 & 811 \\
\hline NGC 1566 & 83 & 140 & 684 & NGC 6812 & 269 & $\cdots$ & $\cdots$ \\
\hline $\begin{array}{l}\text { ESO } \\
\qquad 202-\mathrm{G} 23\end{array}$ & 241 & $\cdots$ & $\cdots$ & ESO 339-G11 & 107 & 284 & $\cdots$ \\
\hline NGC 1808 & 78 & 180 & $\cdots$ & NGC 6860 & 104 & 229 & 1447 \\
\hline $\begin{array}{r}\text { MARK } \\
1210\end{array}$ & 121 & 475 & $\cdots$ & NGC 6890 & 61 & 148 & 354 \\
\hline NGC 2617 & 120 & 1365 & $\cdots$ & NGC 6915 & 168 & $\cdots$ & $\cdots$ \\
\hline $\begin{array}{l}\text { MCG- } \\
\text { 05-23- } \\
004\end{array}$ & 158 & $\cdots$ & $\cdots$ & NGC 6926 & 228 & $\cdots$ & $\cdots$ \\
\hline $\begin{array}{l}\text { MCG- } \\
\text { 05-23- } \\
008\end{array}$ & 211 & $\cdots$ & $\cdots$ & IC 5063 & 154 & 363 & $\cdots$ \\
\hline NGC 2992 & 73 & 204 & $\ldots$ & IC1368 & 72 & 98 & $\cdots$ \\
\hline $\begin{array}{r}\text { MARK } \\
1239\end{array}$ & 148 & 534 & $\cdots$ & NGC 7130 & 70 & 82 & 421 \\
\hline NGC 3100 & 120 & 589 & $\cdots$ & NGC 7213 & 131 & 625 & $\cdots$ \\
\hline IC 2560 & 81 & 212 & $\cdots$ & IC 1459 & 173 & 631 & $\cdots$ \\
\hline $\begin{array}{l}\text { MCG } \\
\quad-06-23- \\
038\end{array}$ & 133 & 312 & $\cdots$ & NGC 7469 & 127 & 229 & 958 \\
\hline $\begin{array}{l}\text { IRAS } \\
\quad 11215\end{array}$ & 66 & 300 & $\cdots$ & NGC 7496 & 50 & 72 & 234 \\
\hline NGC 3783 & 78 & 213 & 1195 & NGC 7591 & 194 & $\cdots$ & $\cdots$ \\
\hline NGC4303 & 52 & 231 & $\cdots$ & NGC 7582 & 64 & 138 & $\cdots$ \\
\hline NGC4404 & 309 & $\cdots$ & $\cdots$ & NGC 7590 & 65 & $\cdots$ & $\cdots$ \\
\hline $3 \mathrm{C} 278$ & 421 & $\cdots$ & $\cdots$ & NGC 7679 & 89 & 397 & $\cdots$ \\
\hline NGC 5506 & 95 & 190 & 426 & NGC 7714 & 66 & 113 & $\cdots$ \\
\hline NGC 5597 & 41 & 109 & $\cdots$ & $\cdots$ & $\cdots$ & $\cdots$ & $\cdots$ \\
\hline
\end{tabular}

Note. Note that these are not corrected for the instrumental width $\left(\sim 45 \mathrm{~km} \mathrm{~s}^{-1}\right)$.

greater and the dust in the coronal emission region is destroyed, allowing the forbidden iron lines to reach such high intensity relative to the hydrogen lines. Systematic changes in width, excitation, and density are consistent with different inner torus radii for the objects shown in Figure 4-MARK 1239 having a small inner torus and MARK 573 having a relatively larger one.

The fact that all our Seyferts lie in the upper part of the Seyfert branch defined by the SDSS galaxies suggests that the band of points that defines the Seyfert sequence on the BPT diagrams is in fact a mixing sequence (presumably a result of aperture effects in the SDSS survey) between ENLR and $\mathrm{H}$ II regions in the SDSS aperture. Such mixing has been investigated in individual galaxies by Scharwächter et al. (2011), Dopita et al. (2014), and Davies et al. (2014a, 2014b). The implication of this is that the Seyfert nuclei and the
Table 4

Measured De-reddened Line Fluxes Relative to $\mathrm{H} \beta=100$

\begin{tabular}{|c|c|c|c|}
\hline Lambda & Line ID & NGC 424 & \\
\hline 3586.3 & {$[\mathrm{Fe} \mathrm{VII}]$} & $20.1 \pm 1.4$ & $\cdots$ \\
\hline 3726,9 & {$\left[\begin{array}{ll}\mathrm{O} & \mathrm{II}\end{array}\right]$} & $113.8 \pm 3.1$ & $\cdots$ \\
\hline 3868.8 & {$[\mathrm{Ne} \quad \mathrm{III}]$} & $92.8 \pm 1.7$ & $\cdots$ \\
\hline 3889 & $\mathrm{H}, \mathrm{He}$ I & $27.1 \pm 2.2$ & $\cdots$ \\
\hline 3967,70 & {$[\mathrm{Ne}$ III] $], \mathrm{H}$} & $44.6 \pm 2.4$ & $\cdots$ \\
\hline 4026.2 & $\mathrm{He}_{\mathrm{I}}$ & $\ldots$ & $\cdots$ \\
\hline 4068,76 & {$[\mathrm{~S}$ III $]$} & $16.5 \pm 0.7$ & $\cdots$ \\
\hline 4101.7 & $\mathrm{H} \delta$ & $31.2 \pm 1.5$ & $\cdots$ \\
\hline 4340.5 & $\mathrm{H} \gamma$ & $50.7 \pm 2.6$ & $\cdots$ \\
\hline 4363.2 & {$\left[\begin{array}{ll}\mathrm{O} & \mathrm{III}\end{array}\right]$} & $37.3 \pm 1.5$ & $\cdots$ \\
\hline 4471.5 & $\mathrm{He}_{\mathrm{I}}$ & $4.1 \pm 0.3$ & $\cdots$ \\
\hline 4685.7 & He II & $32.4 \pm 0.9$ & $\cdots$ \\
\hline 4861.3 & $\mathrm{H} \beta$ & $100.0 \pm 3.2$ & $\cdots$ \\
\hline 4958.9 & {$\left[\begin{array}{ll}\mathrm{O} & \mathrm{III}\end{array}\right]$} & $208.0 \pm 5.5$ & $\cdots$ \\
\hline 5006.8 & {$\left[\begin{array}{ll}\mathrm{O} & \mathrm{III}\end{array}\right]$} & $623.9 \pm 16.4$ & $\cdots$ \\
\hline 5198,200 & {$\left[\mathrm{~N}_{\mathrm{I}}\right]$} & $3.9 \pm 16.7$ & $\cdots$ \\
\hline 5302.6 & [Fe XIV] & $8.1 \pm 0.7$ & $\cdots$ \\
\hline 5411.4 & He I & $2.2 \pm 0.7$ & $\cdots$ \\
\hline 5577.3 & {$\left[\begin{array}{ll}\mathrm{O} & \mathrm{I}\end{array}\right]$} & $\ldots$ & $\cdots$ \\
\hline 5720.7 & {$[\mathrm{Fe}$ VII $]$} & $15.0 \pm 0.8$ & $\cdots$ \\
\hline 5754.9 & {$\left[\begin{array}{ll}\mathrm{N} & \mathrm{II}\end{array}\right]$} & $\cdots$ & $\cdots$ \\
\hline 5875.6 & He I & $10.1 \pm 0.6$ & $\cdots$ \\
\hline 6087.0 & {$[\mathrm{Fe} \mathrm{VII}]$} & $26.4 \pm 1.1$ & $\cdots$ \\
\hline 6300.3 & {$[\mathrm{O}$ I $]$} & $20.7 \pm 8.6$ & $\cdots$ \\
\hline 6363.8 & {$\left[\begin{array}{ll}\mathrm{O} & \mathrm{I}\end{array}\right]$} & $7.0 \pm 0.6$ & $\cdots$ \\
\hline 6374.5 & {$[\mathrm{Fe} \mathrm{X}]$} & $12.1 \pm 0.5$ & $\cdots$ \\
\hline 6547.9 & {$[\mathrm{~N}$ II $]$} & $67.5 \pm 3.1$ & $\cdots$ \\
\hline 6562.8 & $\mathrm{H} \alpha$ & $286.0 \pm 9.0$ & $\cdots$ \\
\hline 6583.2 & {$[\mathrm{~N}$ II $]$} & $202.6 \pm 9.3$ & $\cdots$ \\
\hline 6678.2 & $\mathrm{He}_{\mathrm{I}}$ & $\cdots$ & $\cdots$ \\
\hline 6716.4 & {$\left[\begin{array}{ll}\mathrm{S} & \mathrm{II}\end{array}\right]$} & $26.1 \pm 2.6$ & $\cdots$ \\
\hline 6730.8 & $\mathrm{~S}$ II] & $26.8 \pm 2.6$ & $\cdots$ \\
\hline
\end{tabular}

(This table is available in its entirety in machine-readable form.)

$\mathrm{H}$ II regions associated with them are both of super-solar metallicity.

\subsection{The ENLR and the Circum-nuclear H II Regions}

In Figures 6-8 we present color images of the Seyfert galaxies in our survey. As explained previously, the color scheme was chosen to distinguish Seyferts and their ENLR from $\mathrm{H}$ II regions. This uses the fact that the $\mathrm{H}$ II regions are (at higher metallicities) dominated by $\mathrm{H} \alpha$, while $[\mathrm{N}$ II $] \lambda 6584$ emission is strong and $\left[\mathrm{O}_{\mathrm{III}}\right] \lambda 5007$ is weak. Since $\mathrm{H} \alpha$ is in the red channel and $[\mathrm{N} \mathrm{II}] \lambda 6584$ is in the green channel in these images, $\mathrm{H}_{\text {II }}$ regions appear as either red, orange, or yellowish. In the Seyfert nuclei and their ENLR by contrast, $[\mathrm{O}$ III $] \lambda 5007$ or $\left[\mathrm{N}_{\text {III }} \lambda_{6584}\right.$ are particularly strong. These regions therefore appear violet, blue, turquoise, green, or even white.

Each WiFeS image is $25 \times 37$ arcsec in size; the final row of spaxels is noisy and has been removed. The orientation of the image is shown with a yellow arrow pointing $\mathrm{N}$, and a scale bar $2 \mathrm{kpc}$ long is given. The images are ordered in sequence of increasing R.A.

A few qualitative points can already be made on the basis of these images. First, we see that the conjunction of a Seyfert 

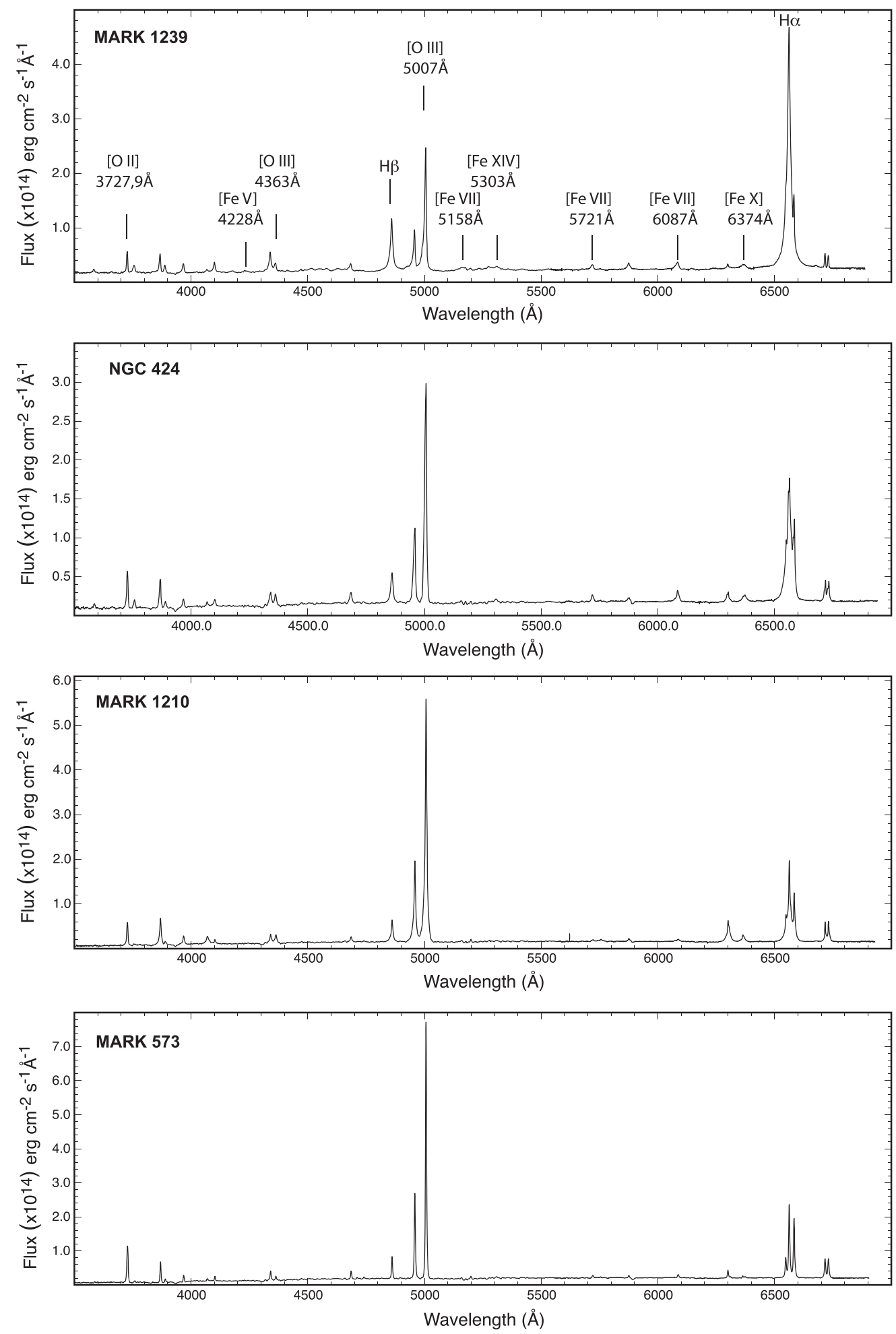

Figure 4. Selection of the S7 galaxy nuclear spectra showing strong coronal-line emission. The main coronal species and a number of the other lines are identified on the first panel. The panels are ordered in terms of the electron density as indicated by the $[\mathrm{O}$ III] $\lambda \lambda 4363 / 5007 \AA$ ratio. MARK 1239 is the densest, with the electron density in the $[\mathrm{O} \mathrm{III}]$ zone exceeding $10^{6} \mathrm{~cm}^{-3}$. Note that this ordering matches the order of the $\mathrm{H} \alpha$ line width and of the $[\mathrm{N} \mathrm{II]} \lambda 6584 / \mathrm{H} \alpha$ ratio. This suggests the existence of correlations between the region of the narrow-line emission, electron density, and the strength of the coronal features.

ENLR lying inside a circumnuclear ring or torus of star formation (typically a few kiloparsecs across) is a fairly common phenomenon. This is clearly seen in 17 galaxies $(\sim 25 \%)$ in the sample. The star-forming ring often shows rapid rotation in the $\mathrm{WiFeS}$ data cube, and such features have been identified as an Inner Lindblad Resonance (Wilson et al. 1993). We will investigate this point in a future paper in this series. Second, where the orientation of the disk can be established, the ionization cones in these cases are arranged in the polar direction, perpendicular to the star-forming ring or torus, 

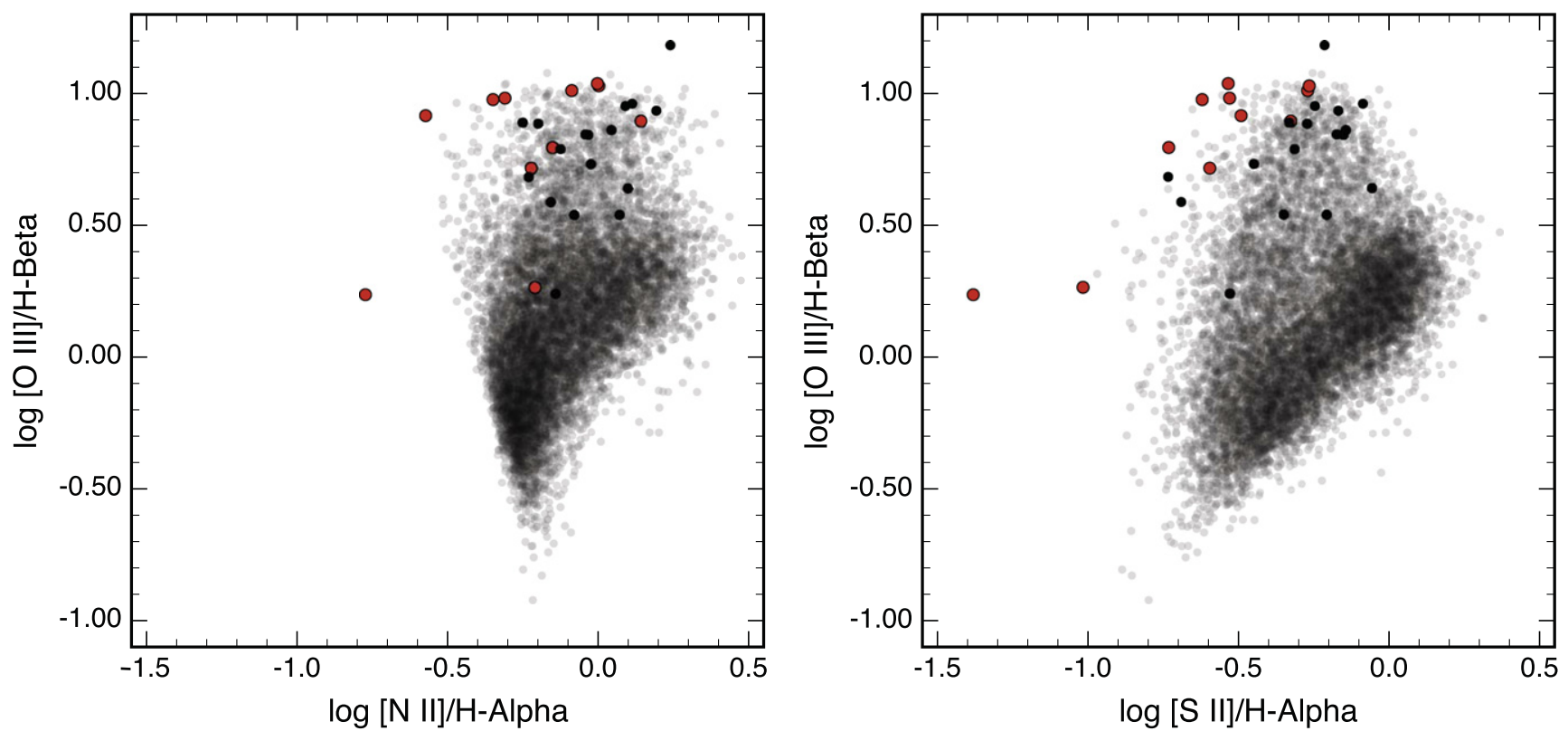

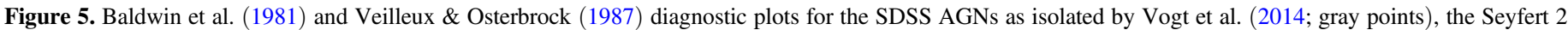

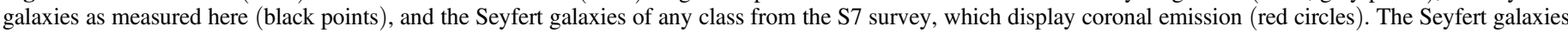

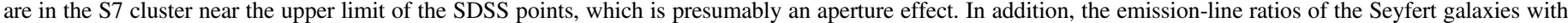
coronal emission are displaced to the left relative to those without.

showing that the inner thick dusty torus of the accretion disk around the black hole must essentially be co-planar, with the star-forming ring located further out. This geometry is seen clearly in nine galaxies of our sample $(\sim 15 \%)$. Third, while all the lower-luminosity AGNs are found to be associated with $\mathrm{H}$ II regions lying within the WiFeS field, many of the higher luminosity objects do not display associated $\mathrm{H}$ II regions, even though the WiFeS field covers a larger area of the galaxy. It is tempting to conclude that in these cases the flux of ionizing photons is sufficiently high as to largely ionize the surrounding ISM and suppress star formation. This is an idea that has been developed by Curran \& Whiting (2013) in an effort to understand why $\mathrm{H}$ I absorption is rare in the hosts of highredshift AGNs. These ideas will be more fully investigated in future papers using the full S7 sample.

\section{NOTES ON INDIVIDUAL OBJECTS}

PKS 0056-572: The object was misclassified in Véron-Cetty \& Véron (2006). We conclude that this object is a QSO at a redshift of $\mathrm{z}=1.46$; see Figure 1 .

NGC424: This Seyfert 2 galaxy was imaged with HST by Malkan et al. (1998). It contains a bright ENLR with relatively broad and strong He II line emission detected out to $\sim 10$ arcsec $(2.2 \mathrm{kpc})$ diameter. A Seyfert 1 nucleus is weakly detected at $\mathrm{H} \alpha$, with prominent coronal-line emission; see Figure 4. Broad $\mathrm{H} \alpha$ and $\mathrm{H} \beta$ have been detected in polarized light from the nucleus by Moran et al. (2000) with a width of $12000 \mathrm{~km} \mathrm{~s}^{-1}$. The nucleus is flanked by two sets of $\mathrm{H}$ II regions within a fastrotating larger star-forming ring.

NGC 613: This galaxy contains a very fine bipolar ENLR $\sim 25 \times 10 \operatorname{arcsec}(\sim 2 \times 0.8 \mathrm{kpc})$. The two lobes of emission are poorly aligned with the radio lobes detected by Condon (1987). The ENLR lobes emerge from a disk of $\mathrm{H}$ II regions, which are strongly dominant close to the nucleus.

IC 1657: Displays a very weak fan-like Seyfert 2 ENLR emerging from one side of a nearly edge-on galaxy disk with many $\mathrm{H}$ II regions. An ENLR ionization cone with an opening angle close to $90^{\circ}$ is visible extending $\sim 11 \operatorname{arcsec}(2.4 \mathrm{kpc})$ from the plane of the galaxy out to the edge of the field. The nucleus displays a strong continuum of old stars, and the emission spectrum is dominated by $\mathrm{H}$ II regions rather than the ENLR.

MARK 573: This Seyfert 2 galaxy ENLR was imaged in [O III] by Schmitt et al. (2003b), revealing a complex knotty ionization cone on a $\sim 10 \operatorname{arcsec}(\sim 2.2 \mathrm{kpc})$ scale. The WiFes data shows a very bright and much more extensive barrelshaped ENLR that is more than $8 \mathrm{kpc}$ long. There is no sign of embedded $\mathrm{H}$ II regions. The nuclear spectrum shows strong coronal emission; see Figure 4. Fischer et al. (2010) using WFPC2 and STIS observations argue that the ionizing radiation field, confined to an ionization cone from the central engine, is inclined and intersects and ionizes the inner spiral arms of the galaxy, driving these into outflow.

IRAS 01475-0740: The nucleus of this Seyfert 2 galaxy is very bright, but the ENLR can be traced in the WiFeS data cube over some $\sim 10 \times 7 \operatorname{arcsec}(\sim 3.3 \times 2.3 \mathrm{kpc})$. The $H S T$ [O III] image by Schmitt et al. (2003a) is dominated by the compact nucleus. Broad lines have been detected in polarized emission (Tran 2003), showing that the AGN core of the galaxy is heavily obscured.

$N G C$ 833: This LINER galaxy is tidally interacting with NGC 835, and these are two members of the interesting and highly active Hickson Compact Group 16. All galaxies in this group are either active or starburst. The equivalent width of the $\left[\mathrm{N}\right.$ II] lines is quite high, and the $\left[\mathrm{O}_{\mathrm{III}}\right]$ and $\left[\mathrm{O}_{\mathrm{I}}\right]$ lines are quite strong, superimposed on a strong old star continuum.

$N G C$ 835: This Seyfert 2 galaxy is tidally interacting with NGC 833 and displays a long tidal tail. The nuclear spectrum is dominated by a strong post-starburst continuum with deep $\mathrm{NaD}$ absorption. In X-rays, Turner et al. (2001) confirmed the presence of an AGN in both NGC 835 and NGC 833. The ENLR, $\sim 8 \times 5 \operatorname{arcsec}(\sim 1.9 \times 1.3 \mathrm{kpc})$, is contained within an 


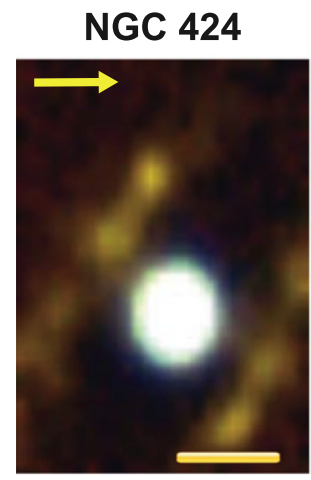

Sy 241.67

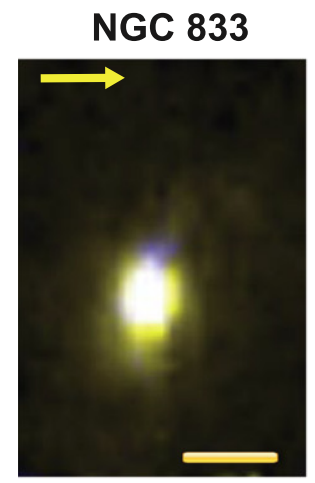

LINER $\mathbf{3 9 . 3 3}$

NGC 1125

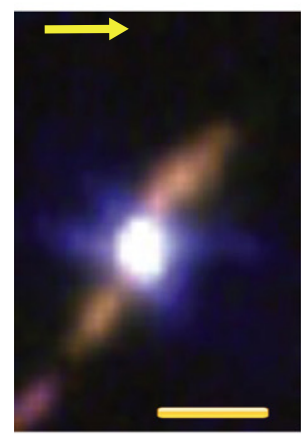

Sy 241.50

MARK 1210

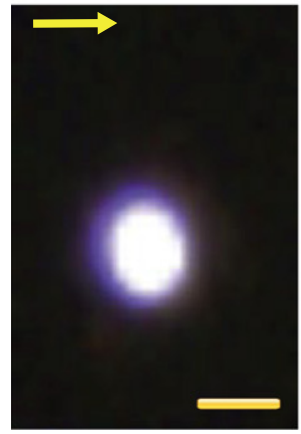

Sy 241.83

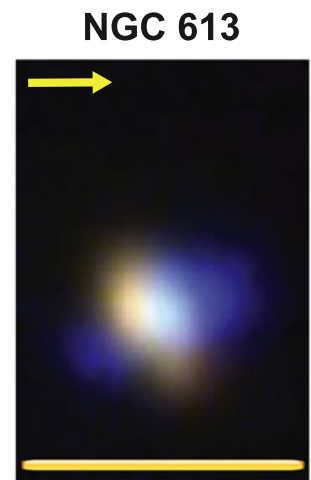

Sy 239.99

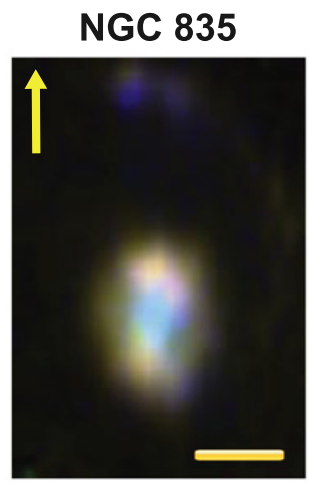

Sy 240.06

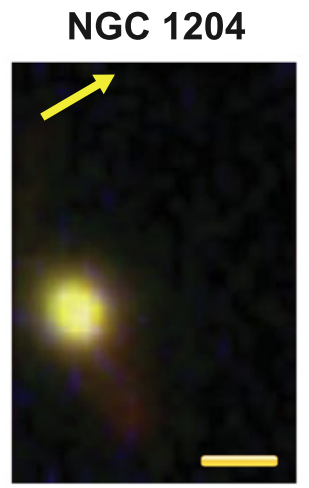

SB $\quad 40.55$

NGC 2617

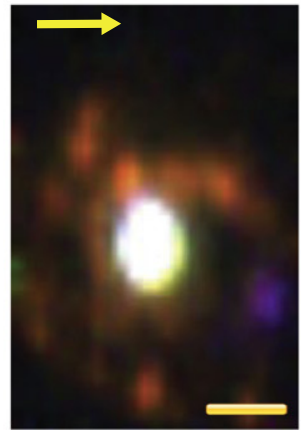

Sy $1 \quad 41.23$

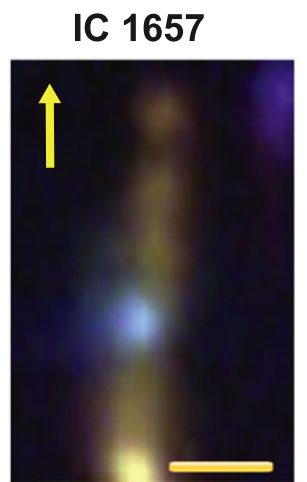

Sy 239.93

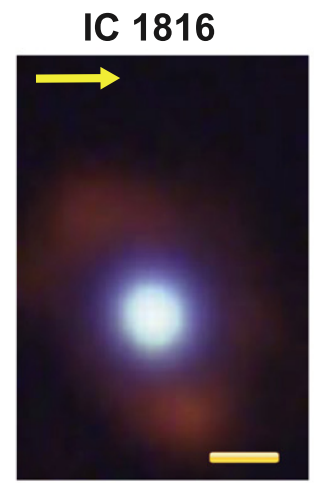

Sy 241.25

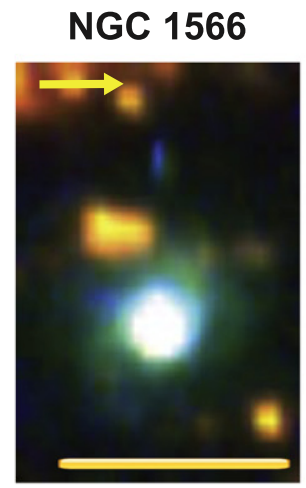

Sy 240.01

MCG-01-24-012

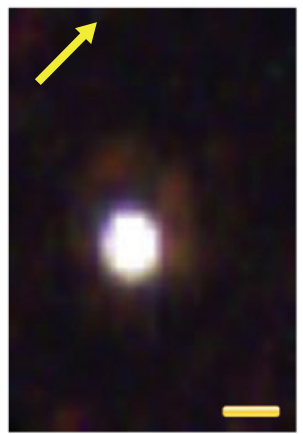

Sy 2

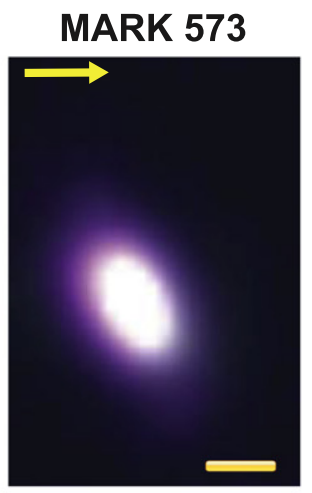

Sy 241.72

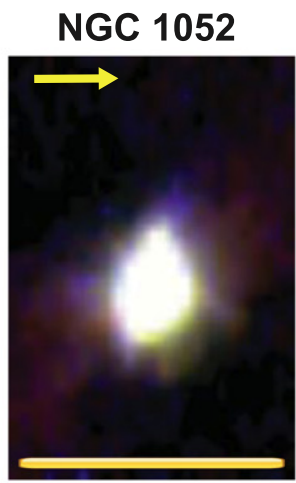

LINER $\mathbf{3 8 . 6 2}$
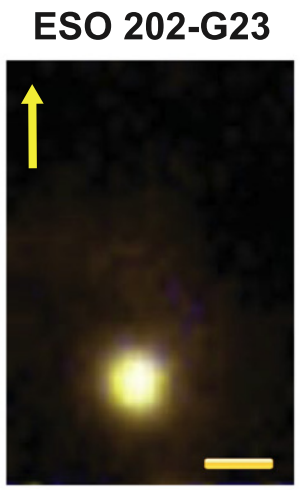

LINER $\quad 40.30$

MCG-05-23-004

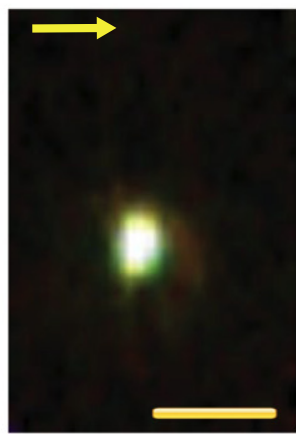

LINER $\quad 39.01$

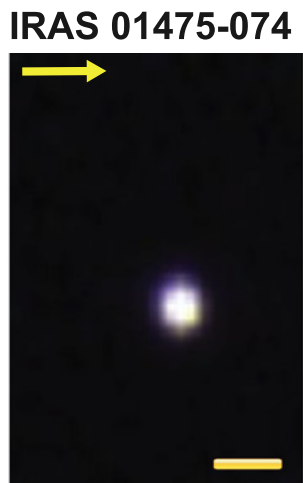

Sy 241.73

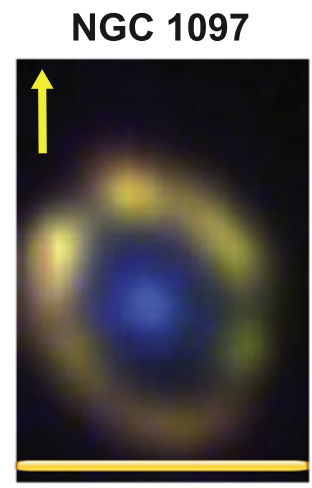

LINER $\mathbf{3 8 . 5 5}$

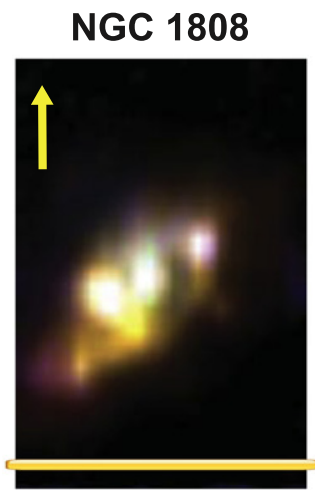

SB $\quad 39.89$

MCG-05-23-008

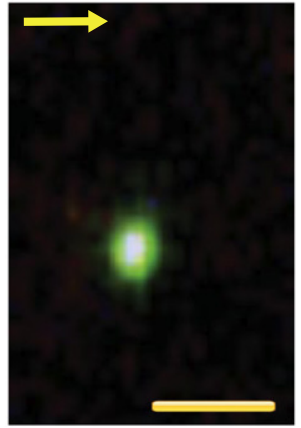

LINER $\mathbf{3 8 . 8 3}$

Figure 6. Color images of the galaxies lying between $\mathrm{RA}=00 \mathrm{Hr}$ and $09 \mathrm{Hr} 44 \mathrm{~m}$. All images are $25 \times 37$ arcsec in size. The direction of north is indicated by a yellow arrow. The galaxy type and nuclear [O III] $\lambda 5007$ luminosity is given below each image. In these images $\mathrm{H} \alpha$ is in the red channel, [N II] $\lambda 6584$ is in the green, and [O III] $\lambda 5007$ in the blue channel to distinguish the $\mathrm{H}$ II regions (gold, yellow, or red) from the Seyfert and LINER nuclei and their ENLR (blue, green, turquoise, or violet). 


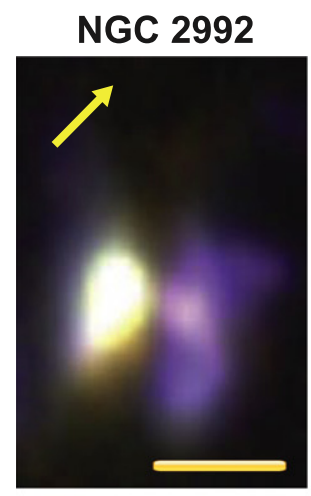

Sy $2 \quad 41.77$

IRAS 11215-2806

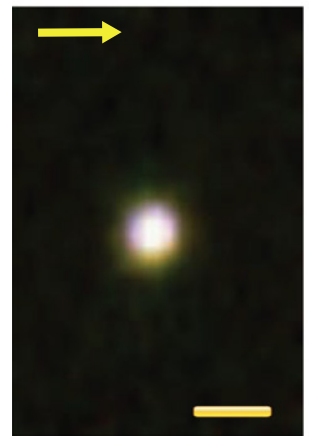

Sy $2 \quad 40.87$

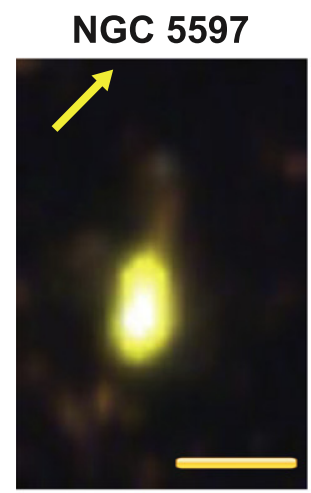

SB 40.31

ESO 137-G34

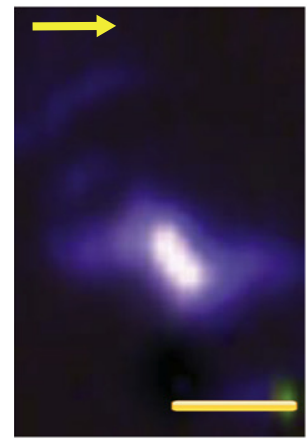

Sy2 41.66
MARK 1239

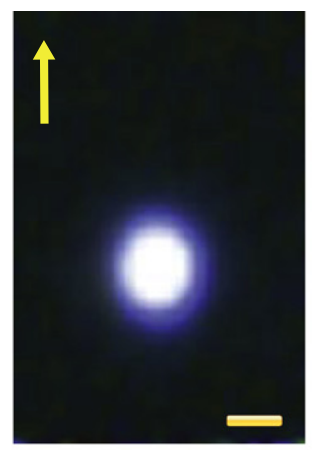

Sy $1 \quad 42.47$

NGC 3783

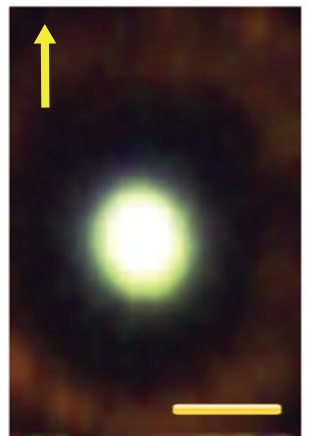

Sy 141.12

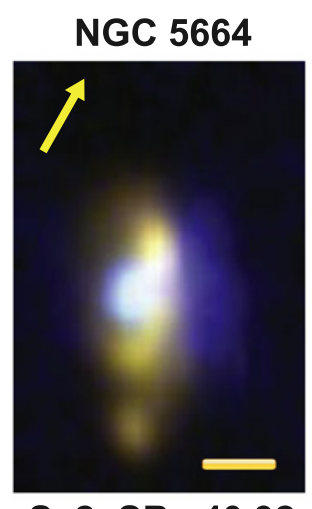

Sy2+SB 40.92
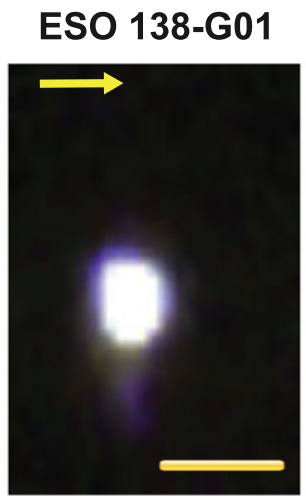

Sy2 41.93

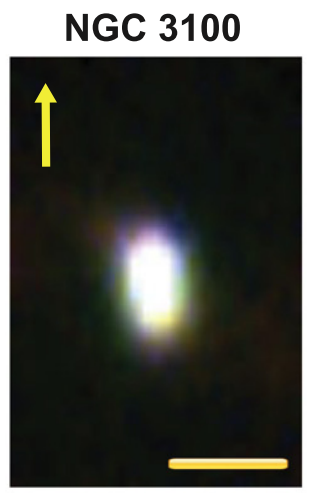

LINER 39.23

NGC 4303

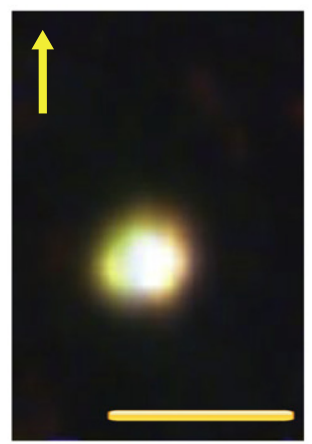

LINER/SB $\mathbf{4 0 . 0 2}$

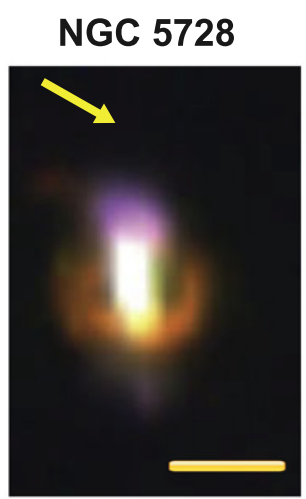

Sy $2 \quad 41.38$

NGC 6221

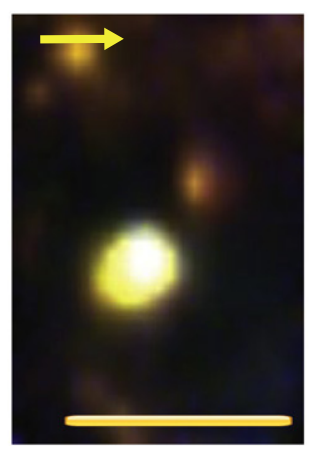

Sy2+SB $\quad \mathbf{4 0 . 8 9}$

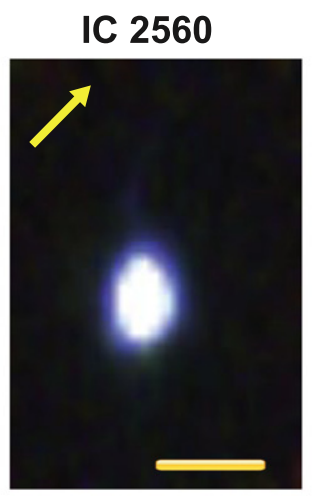

Sy 241.35

NGC 4404

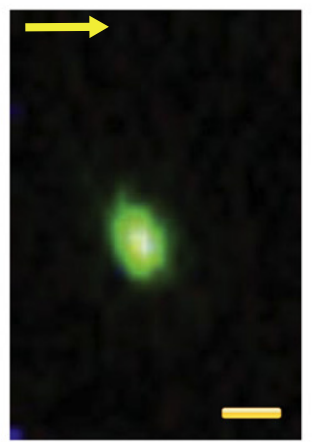

LINER 39.42

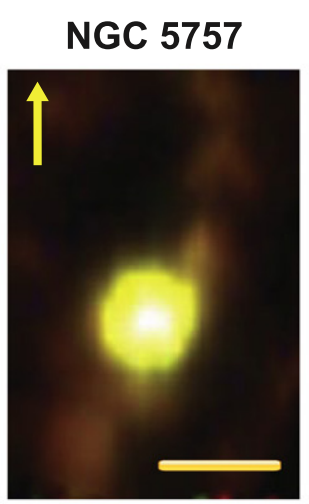

SB 39.91

NGC 6300

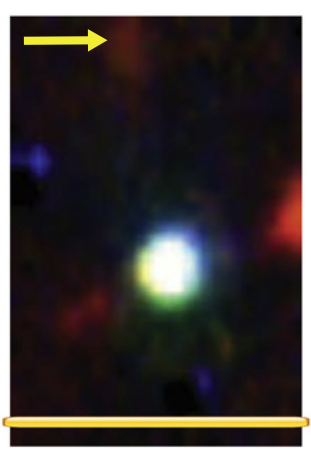

Sy2 40.99

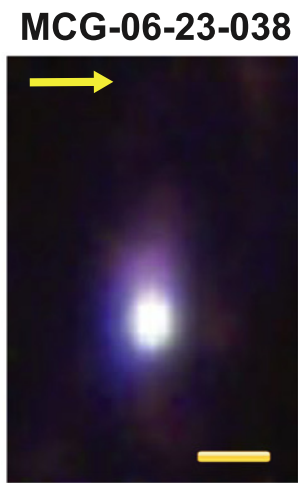

Sy 241.41

NGC 5506

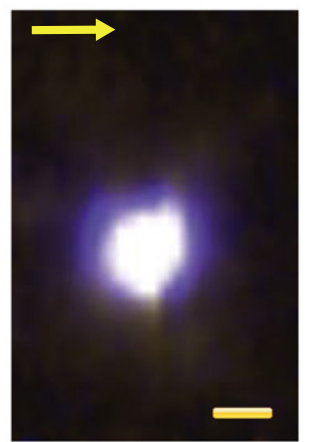

Sy 241.44

NGC 6000

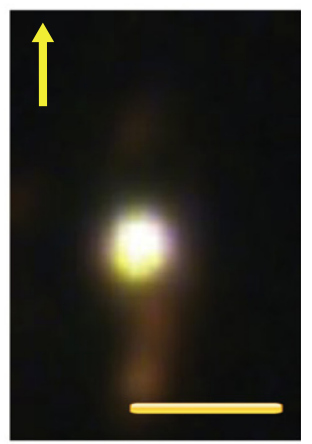

SB $\quad 40.28$

FAIRALL 49

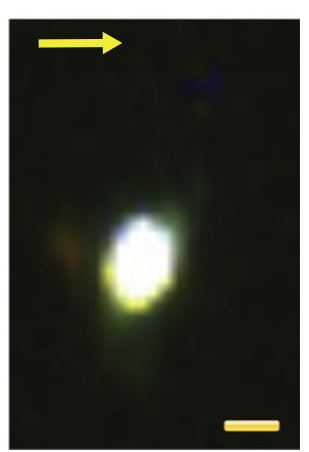

Sy2 42.68

Figure 7. As Figure 6, but for the galaxies lying between R.A. $=09 \mathrm{hr} 45$ minutes and $18 \mathrm{hr} 37$ minutes. 


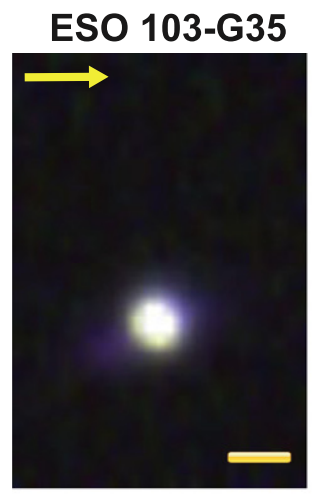

Sy $2 \quad 41.54$

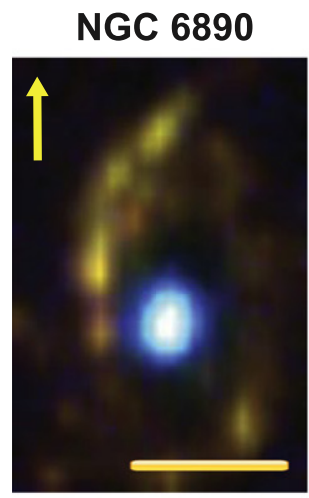

Sy $2 \quad 40.87$

NGC 7130

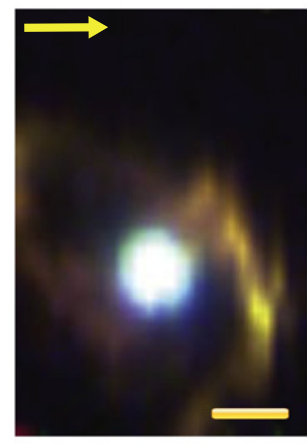

SB+Sy2 41.66

NGC 7582

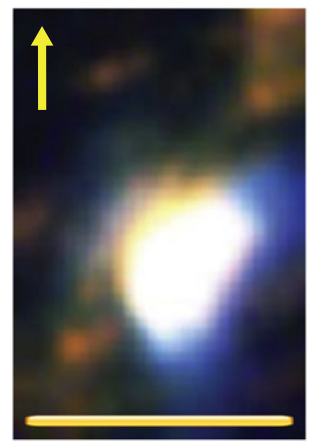

Sy2 + SB $\quad 40.43$
FAIRALL 51

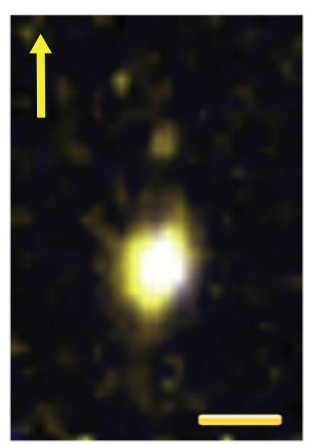

Sy $1 \quad 40.83$

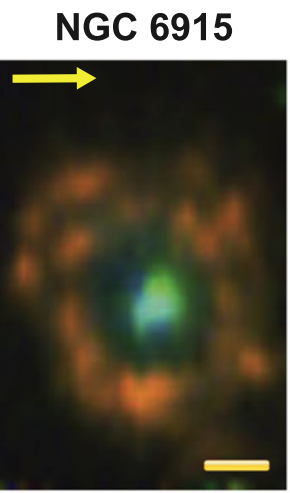

LINER 39.34

NGC 7213

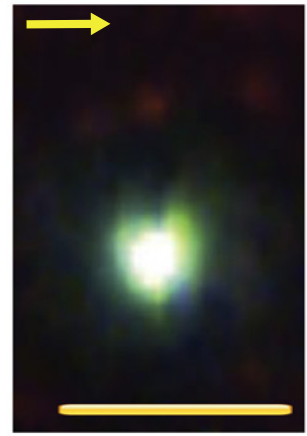

LINER 39.72

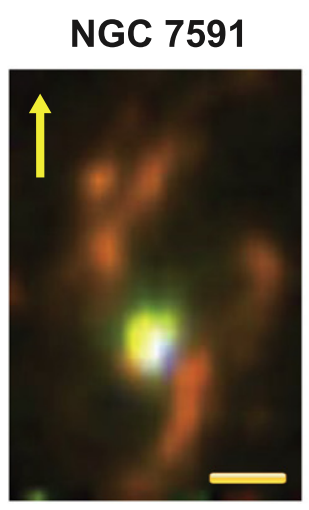

LINER $\quad \mathbf{4 0 . 3 7}$

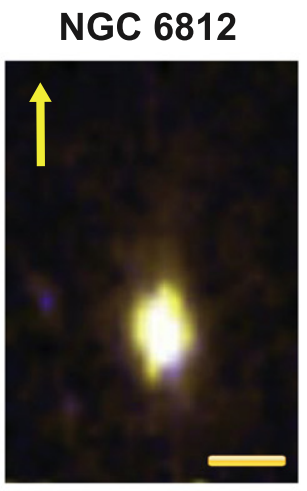

LINER 39.66

NGC 6926

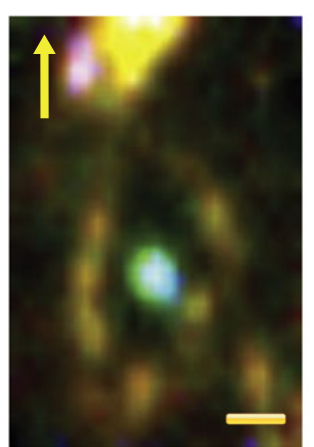

Sy 241.22

IC 1459

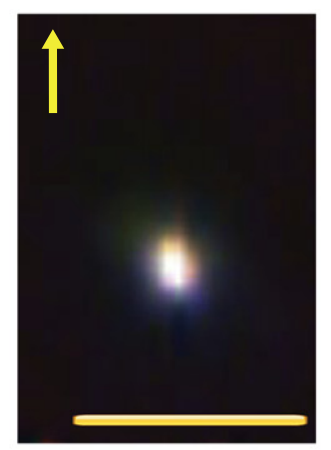

LINER 39.09

NGC 7590

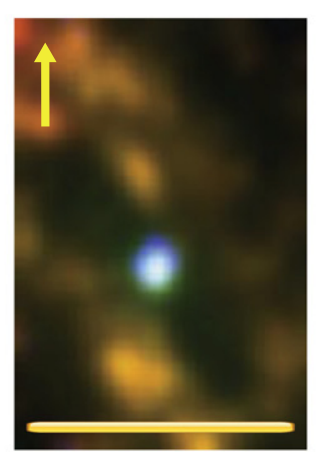

Sy2 39.75

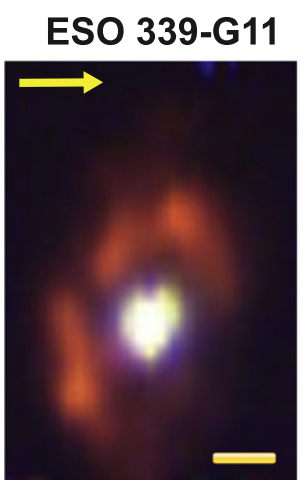

Sy 242.24

IC 5063

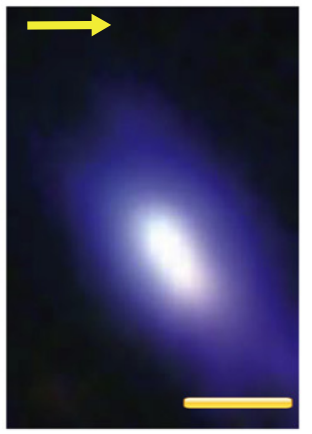

Sy 241.77

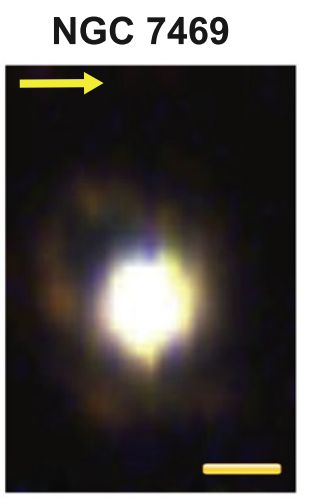

Sy 142.77

NGC 7679

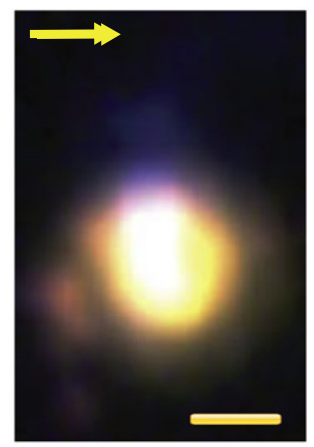

Sy2 + SB 40.98
NGC 6860

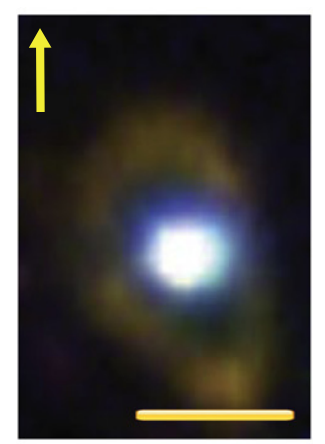

Sy $1 \quad 40.83$

IC 1368

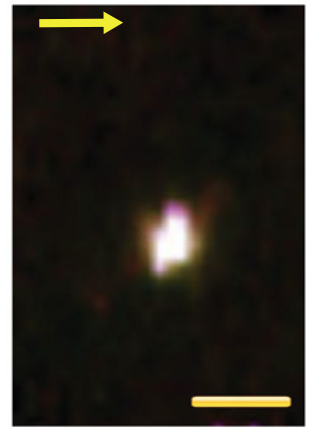

Sy 241.49

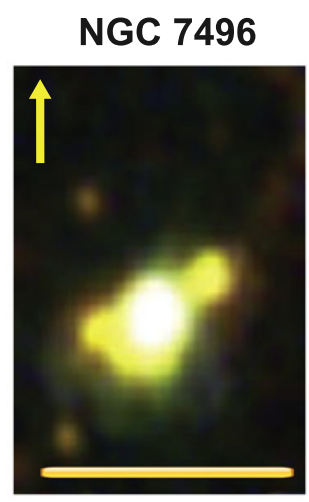

Sy $2+$ SB 39.88

NGC 7714

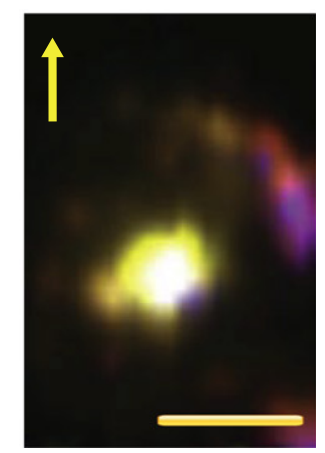

SB 41.38

Figure 8. As Figures 6 and 7, but for the galaxies lying between RA $=18 \mathrm{hr} 38$ minutes and $24 \mathrm{hr}$. 
elliptical starburst ring $\sim 14 \times 9$ arcsec $(\sim 3.4 \times 2.3 \mathrm{kpc})$ in diameter.

IC 1816: This Seyfert 2 galaxy with a bar + ring structure and tidally disturbed outer arms (Malkan et al. 1998). The ENLR has been studied by Fehmers et al. (1994) and Cid Fernandes et al. (1998), and long slit spectroscopy obtained by Fraquelli et al. (2003). The WiFeS nuclear spectrum displays fairly broad forbidden lines and weak coronal lines; [Fe VII] 6087, 5721 A lines are visible. The ENLR fills the region within the prominent star-forming ring located at a radial distance of $\sim 3 \mathrm{kpc}$.

$N G C$ 1052: This classical liner shows an X-shaped cross of strong [O III] emission and an extended ridge of $\mathrm{H} \alpha$ emission. A detailed accretion plus jet cocoon shock model for the excitation of this complex source will be presented in the next paper of this series (Dopita et al. 2015, in press)

NGC1097: A tidally distorted grant-design spiral with a weak active nucleus embedded in bright stellar continuum, surrounded by a star-forming ring with $\mathrm{E}+\mathrm{A}$ spectrum. Broad $\mathrm{NaD}$ absorption is evident around the nucleus. The nucleus displays a high velocity dispersion with very strong $[\mathrm{N} \mathrm{II}]$. A faint ENLR reappears outside the star-forming ring. A study by Fathi et al. (2006) using the GMOS-IFU and high-resolution HST-ACS observations shows that the nuclear activity appears to be fed by radial flow from the star-forming ring toward the nucleus. The nuclear activity is variable, and the galaxy has been classified as a Seyfert 1 on the basis of broad $\mathrm{H} \alpha$ when more active (Storchi-Bergmann et al. 1997, 2003).

NGC 1125: This Seyfert 2 galaxy contains a superb ionization cone extending not quite at right angles from the disk, which is seen nearly edge on. The ENLR appears as a double paraboloidal structure and is dynamically quiescent, corotating with the disk gas. The orientation of the ENLR is well aligned with the much larger $\sim 12 \mathrm{kpc}$ double-lobe structure seen at $8.4 \mathrm{GHz}$ (Thean et al. 2000). There are a number of bright $\mathrm{H}$ II regions in the disk. An HST F606W continuum image was published by Malkan et al. (1998). Mulchaey et al. (1996a) find that the [O III] emission is only slightly resolved with $H S T$ - presumably due to surface brightness limitations.

NGC 1204: Appears to be a fairly metal-rich starburst. The nuclear spectrum shows a strong, heavily reddened late- or post-starburst continuum. Zaw et al. (2009) note that the line ratios in this object put it in the Kewley et al. (2006) transition zone, suggesting that shocks may be important in powering this source. A broad component to $\mathrm{H} \alpha$ is detected at the nucleus.

$N G C$ 1566: A superb two-arm spiral with very active star formation, this Seyfert 1 galaxy has an ENLR $\sim 15 \times 10$ arcsec $(\sim 1.5 \times 1.0 \mathrm{kpc})$ extended in the NE-SW direction. The circumnuclear reddening is high, so the ENLR is more easily traced in $\left[\mathrm{N}_{\mathrm{II}}\right] \mathrm{A}$ " broad component to $\mathrm{H} \alpha$ as detected at the nucleus, confirming the Seyfert 1 type given on the basis of HST FOS observations (Kriss et al. 1991).

ESO 103-G35: The nuclear emission-line spectrum suggests that this object is a very heavily reddened Seyfert 2, or possibly a LINER with very strong $[\mathrm{N}$ II $]$ and $\left[\mathrm{O}_{\mathrm{I}}\right]$. The underlying continuum is also very red. The disturbed morphology of the galaxy suggests that it is a post-merger.

$N G C$ 1808: This galaxy is simply a circumnuclear $(\sim 1 \mathrm{kpc})$ starburst galaxy with very deep $\mathrm{NaD}$ absorption, and a latestarburst continuum spectrum. It is hard to understand why it was ever assigned a Seyfert 2 class, but the Véron-Cetty \& Véron (2006) catalog now has it as an H2 type.
MARK 1210: This galaxy has been imaged with HST in the F606W filter by Malkan et al. (1998). All the emission lines in this Seyfert 2 galaxy have a broad $\left(\sim 475 \mathrm{~km} \mathrm{~s}^{-1}\right)$ component. The ENLR is confined within the central $\sim 1.0 \mathrm{kpc}$, which is in agreement with the IR results of Mazzalay \& Rodríguez-Ardila (2007). The coronal lines are strong, see Figure 4, and the IR coronal spectrum has been extensively studied by Mazzalay \& Rodríguez-Ardila (2007). Coronal lines of [S VIII], [S IX], [Si VII], [Si X], and [Ca VIII] were detected.

NGC 2617: This Seyfert 1 galaxy displays extremely broad Balmer line emission with a line profile that is steeper on the blue side. By contrast the ENLR lines are relatively narrow $\left(\sim 120 \mathrm{~km} \mathrm{~s}^{-1}\right)$. The ENLR is elliptical $\sim 9 \times 6$ arcsec $(\sim 2.6 \times 1.7 \mathrm{kpc})$ and orientated almost E-W, but there is a prominent $\mathrm{ENLR} / \mathrm{H}$ II region knot at $\mathrm{PA}=330^{\circ}$ and at 15 arcsec from the nucleus $(\sim 4.4 \mathrm{kpc})$. The nucleus is surrounded by many bright $\mathrm{H}$ II regions.

MCG-01-24-012: This is a Seyfert 2 galaxy detected in hard $\mathrm{X}$-rays by BeppoSAX by Malizia et al. (2002). It was imaged using HST by Schmitt et al. (2003a), who found the ENLR to be extended by $880 \times 440 \mathrm{pc}$ at PA $=750^{\circ}$. In the WiFeS data cube, the ENLR can be traced further, out to $3.7 \times 2.5 \mathrm{kpc}$, with a major axis agreeing with that measured with $H S T$. The ENLR is surrounded by a faint ring of $\mathrm{H}$ in regions, about $3 \mathrm{kpc}$ in radius.

MCG-05-23-004: This poorly studied late-type galaxy is a LINER with LINER-like spiral emission extensions. These can be traced out to $\sim 12 \operatorname{arcsec}(2.3 \mathrm{kpc})$ from the nucleus.

MCG-0105-23-008: This is also a faint LINER in an Elliptical galaxy.

$N G C$ 2992: This is a tidally distorted galaxy interacting strongly with NGC 2993. The very extensive ENLR has been studied and interpreted through multi-slit spectroscopy by Allen et al. (1999). The galaxy displays an extensive biconical ENLR with a large opening angle extending above and below a nearly edge-on dusty disk. The nucleus exhibits variability, with a Seyfert 1 nucleus appearing and fading, apparently correlated with X-ray activity. The original spectra by Ward et al. (1980) showed broad wings to $\mathrm{H} \alpha$, while in 1997 they had disappeared (Allen et al. 1999). By 2000 they had returned (Gilli et al. 2000), but then once again disappeared in the observations by Trippe et al. (2008), who argue against the hypothesis that these variations are simply due to variable extinction in the circumnuclear dust lane seen with HST (Malkan et al. 1998). In our spectrum, we once again see broad wings to $\mathrm{H} \alpha$. These wings were detected in polarized light (Lumsden et al. 2004).

MARK 1239: This E-S0 galaxy contains a very luminous Seyfert 1 with a very rich and high-excitation coronal spectrum, including [Fe XIV]; see Figure 4. The ENLR is very bright and extensive with an elliptical morphology $(\sim 17 \times 15$ arcsec, $\sim 7.1 \times 6.3 \mathrm{kpc})$. There is no sign of any $\mathrm{H}$ II regions.

$N G C$ 3100: This is a LINER in a SO galaxy with-in common with other LINERS - very low extinction, but deep $\mathrm{NaD}$ absorption.

IC 2560: A Seyfert 2 galaxy with relatively narrow lines and coronal emission. There is a hint of a broad component to $\mathrm{H} \alpha$. The ENLR is very extensive $(\sim 18 \times 13$ arcsec, $\sim 3.8 \times 2.8$ kpc) with a jet-like extension toward the $\mathrm{N}$.

MCG-06-23-038: The ENLR $(\sim 11 \times 9$ arcsec, $\sim 3.5 \times 2.9$ $\mathrm{kpc})$ is dynamically active, showing pronounced line splitting. The ENLR is embedded in a rotating disk of $\mathrm{H}$ II regions 
elongated along the major axis of the galaxy. There are a number of other $\mathrm{H}$ II regions scattered across the field, which are associated with the outer stellar ring.

IRAS 11215-2806: This Seyfert 2 galaxy has a fairly compact ENLR $(\sim 7 \times 7$ arcsec, $\sim 2.1 \mathrm{kpc})$. It has been imaged with HST in the F606W filter by Malkan et al. (1998).

NGC 3783: This barred ring spiral was imaged with HST in the continuum by Malkan et al. (1998), and the Seyfert 1 nucleus in $[\mathrm{O}$ III] by Schmitt et al. (2003a). However, the [O III] is almost point-like in the HST image. The measured [O III] luminosity $\log L=41.08$ agrees closely with our measurement: $\log L=41.12$. We find this compact nucleus, strong in coronal-line emission, to be surrounded by an extensive low surface brightness ENLR $\sim 18$ arcsec, or $\sim 3.8 \mathrm{kpc}$ in diameter, effectively filling the central region out to the star-forming ring, marked by $\mathrm{H}$ in regions.

$N G C$ 4303: The nuclear spectrum is that of a late starburst, or possibly a LINER type. However, $\left[\mathrm{O}_{\mathrm{I}}\right] \lambda 6300$ is very weak for a LINER. The nucleus is surrounded by a star-forming ring $\sim 9 \operatorname{arcsec}(1.1 \mathrm{kpc})$ in diameter (Mazzuca et al. 2008), and many $\mathrm{H}$ II regions are scattered across the field-c.f., $\mathrm{H} \alpha$ image from Banfi et al. (1993).

$N G C$ 4404: This is a very weak and strongly reddened LINER, apparently in interaction with NGC 4403.

3 C 278: This object, VV 201, is a closely interacting double early type galaxy and a very strong radio source. No emission is visible at the position of the double-lobe radio source nucleus (Morganti et al. 1993). The spectrum is simply that of an Elliptical galaxy.

NGC 5506: This disk galaxy is seen nearly edge-on, and a strong lane of absorption crosses the nucleus on the F606W HST image of Malkan et al. (1998). The WiFeS nuclear spectrum of this Seyfert 2 galaxy shows weak coronal emission. Nagar et al. (2002) classify it as an obscured narrow-line Seyfert 1 on the basis of a near-IR spectrum. The ENLR is elongated in the NE direction at high flux levels in agreement with the IR measurements by Raban et al. (2008), but at low flux levels it appears more as a double fan with wide opening angle orientated $\mathrm{N}-\mathrm{S}$ at right angles to the stellar disk. Its full extent is $\sim 15 \times 12$ arcsec or $\sim 2.1 \times 1.6 \mathrm{kpc}$.

NGC 5597: This somewhat tidally disturbed galaxy forms an interacting physical pair with NGC 5595. The nuclear region forms a bar of star formation imaged with HST in the red continuum by Malkan et al. (1998). The nuclear continuum spectrum is dominated by young stars, and a broad He II $\lambda 4686$ feature from a WR population is faintly visible. A number of other $\mathrm{H}$ II regions are visible in the general field.

NGC 5664: This poorly studied Seyfert 2 is embedded in a star-forming ring $\sim 13 \operatorname{arcsec}(4.0 \mathrm{kpc})$ in diameter. It displays a magnificent bi-cone ENLR orientated in the polar direction of this disk, with an opening angle of $90^{\circ}$. This is much fainter on the east side. The ENLR extends right across the WiFeS FOV and is therefore larger than $\sim 25 \operatorname{arcsec}(7.7 \mathrm{kpc})$ across. The ENLR is co-rotating with the galaxy, and is dynamically quiescent.

NGC 5728: A barred ring spiral galaxy with a Seyfert 2 nucleus, it displays a well-collimated bright two-sided ENLR first described by Wilson et al. (1993) on the basis of HST images. The ENLR emerging SE-NW at right angles to a starforming ring with a diameter of $\sim 2 \mathrm{kpc}$. The length of the ENLR is $\sim 22 \operatorname{arcsec}(4.4 \mathrm{kpc})$ across-much larger than the $1.8 \mathrm{kpc}$ reported by Wilson et al. (1993). The inner ENLR has strong line splitting in $\left[\mathrm{O}_{\mathrm{III}}\right] \lambda 5007$. The nuclear spectrum shows a Seyfert 2 spectrum superimposed on a continuum spectrum showing signs of recent star formation. The $[\mathrm{Fe} \mathrm{VII}] \lambda$ 6087 is present, but is very weak compared to the continuum. In the IR the coronal region has been detected in [Ca VIII] (Emsellem et al. 2001) and in its [Si VI] emission (Riffel et al. 2006).

NGC 5757: This barred spiral galaxy has a nuclear starburst with low reddening and high metallicity (Saraiva et al. 2001) embedded in spiral arms crowded with $\mathrm{H}$ II regions. It has been imaged with HST in the continuum by Malkan et al. (1998).

NGC 6000: A nuclear starburst on a barred spiral, very similar to NGC 5757, but with deep $\mathrm{NaD}$ absorption. It has been imaged using WFPC2 in the F606W filter with HST by Carollo et al. (1997).

ESO 137-G34: A spectacular Seyfert 2 with a pronounced line splitting, see Figure 3 , and a very extensive biconical ENLR with an opening angle of $80^{\circ}$ and containing multiple shells. The ENLR extends beyond the WiFeS field, and is therefore in excess of $40 \operatorname{arcsec}(7.4 \mathrm{kpc})$ in diameter. The nucleus has been imaged with $H S T$ in the red continuum by Malkan et al. (1998), and the ENLR has been studied with HST by Mulchaey et al. (1996a).

ESO 138-G01: A luminous Seyfert 2 with a rich coronal-line spectrum. There is a suggestion of a broad-line component underlying $\mathrm{H} \alpha$. The ENLR of this galaxy has been imaged by Schmitt \& Storchi-Bergmann (1995), who traced the ENLR over about 16 arcsec, out to the inner jet region visible in Figure 7. However, in the WiFeS data cube, we see the ENLR extending over the full field, out to at least $35 \operatorname{arcsec}(6.4 \mathrm{kpc})$ in diameter. In particular, there are two knots of emission at $\sim 15$ arcsec to either side of the nucleus at PA $=105^{\circ}$. Both show pronounced line splitting with $\Delta v \sim 350 \mathrm{~km} \mathrm{~s}^{-1}$.

$N G C$ 6221: This galaxy, imaged in $\mathrm{H} \alpha$ by Ryder \& Dopita (1993) and in the optical and IR at high resolution by Levenson et al. (2001), and also studied spectroscopically by $\mathrm{Gu}$ et al. (2006), contains a very weak Seyfert 2 nucleus (detected by the broader $[\mathrm{O}$ III $] \lambda 5007$ line) embedded in a bright starburst nucleus. This galaxy is an X-ray-loud composite galaxy, with very weak AGN activity seen at optical or IR wavelengths, but displaying strong X-ray emission. Obscuration by a column of $N_{H}=10^{22} \mathrm{~cm}^{2}$ is sufficient to explain these properties (Levenson et al. 2001). Many $\mathrm{H}$ in region complexes are scattered across the WiFeS field of view.

NGC 6300: This barred ring spiral galaxy has a heavily obscured Seyfert 2 nucleus with deep $\mathrm{NaD}$ absorption. Long slit spectroscopy obtained by Fraquelli et al. (2003). This galaxy changes from Compton-thick to Compton-thin at X-ray wavelengths over timescales of a few years (Matt et al. 2003), and contains an $\mathrm{H}_{2} \mathrm{O}$ maser (Greenhill et al. 2003). The ENLR is approximately $7 \times 5$ arcsec, or $0.5 \times 0.3 \mathrm{kpc}$ in diameter in [O III $\lambda 5007$, but can be traced further out in the NE-SW axis in $[\mathrm{N}$ II $] \lambda 6584$. A number of $\mathrm{H}$ II region complexes are scattered across the WiFeS field of view.

FAIRALL 49: This ultra luminous IR galaxy with a compact nucleus (Malkan et al. 1998) is a reddened Seyfert 2 with fairly broad forbidden lines and weak coronal lines of [Fe VII] 6087, $5721 \AA$. The ENLR can be traced across much of the field $(\sim 28 \times 10$ arcsec, $\sim 11 \times 4 \mathrm{kpc})$. Broad $\mathrm{H} \alpha$ and $\mathrm{H} \beta$ components are detected in the nuclear spectrum. These components have also been detected in polarized light (Lumsden et al. 2004). 
ESO 103-G35: A Seyfert 2 galaxy studied by $\mathrm{Gu}$ et al. (2006) with an extensive clumpy bipolar ENLR with an opening angle of $\sim 45^{\circ}$ orientated at $\mathrm{PA}=25^{\circ}$ (roughly in the polar direction of the S0 host galaxy) and extending across the field of view of WiFeS $(27 \operatorname{arcsec} 10 \mathrm{kpc})$. Away from the nucleus the ENLR lines are narrow and dynamically inactive.

FAIRALL 51: A Seyfert 1 with broad-line components visible in $\mathrm{He} \mathrm{I}, \mathrm{He} \mathrm{II}$, and down to $\mathrm{H} \delta$. The nuclear spectrum displays a strong high-excitation intermediate-width coronal spectrum with $[\mathrm{Fe} \mathrm{XIV]} 5303 \AA$ and $[\mathrm{Fe} \mathrm{X}] 6374 \AA$ lines prominent. Other forbidden lines are narrower. The continuum of this galaxy is highly polarized (Smith et al. 2004). The ENLR can be traced over $\sim 10 \times 7$ arcsec, $\sim 2.8 \times 1.9 \mathrm{kpc}$. A few faint $\mathrm{H}$ il regions are also visible in the field.

NGC 6812: A classical LINER galaxy with E-type continuum and deep $\mathrm{NaD}$ absorption. This galaxy is in a group of three and may be interacting. The emission-line region is elongated in the $\mathrm{N}-\mathrm{S}$ direction (the polar direction in the galaxy), and has dimensions $\sim 24 \times 13$ arcsec, $\sim 7.2 \times 3.9$ kpc. Its velocity structure suggests that it is probably an outflow.

ESO 339-G11: A luminous IR Sb galaxy with a Seyfert 2 nucleus displaying strong $[\mathrm{N}$ II] and weak coronal lines on a reddened, late-type stellar spectrum. The $\sim 9 \times 5$ arcsec $(\sim 3.3 \times 1.8 \mathrm{kpc})$ ENLR is located within a $\sim 3.5 \mathrm{kpc}$ radius rapidly rotating starburst ring of $\mathrm{H}$ II regions.

NGC 6860: The spiral host galaxy is classified as a LIRG. The Seyfert 1 nucleus is surrounded by ENLR $\sim 15 \operatorname{arcsec}$ $(4.3 \mathrm{kpc})$ in diameter surrounded by a distorted ring of offnuclear $\mathrm{H}$ II regions with A-type absorption lines. This galaxy has been studied by high spatial resolution optical imaging and optical and near-IR spectroscopy by Lipari et al. (1993). The inner ENLR has been imaged in $[\mathrm{O}$ III $] \lambda 5007$ by Schmitt et al. (2003a).

NGC 6890: This SA ringed spiral has a nucleus of Seyfert 2 type with coronal emission. Long slit spectroscopy was obtained by Fraquelli et al. (2003), and the high-excitation, mid-IR lines were investigated by Pereira-Santaella et al. (2010). It contains a bright region of broad $[\mathrm{O}$ III $] \lambda 5007$ in an ENLR $\sim 8 \times 8 \operatorname{arcsec}(1.2 \mathrm{kpc})$, inside a bright and complex elliptical star-forming ring with major axis 28 arcsec $(4.2 \mathrm{kpc})$ with many bright $\mathrm{H}$ in regions.

NGC 6915: This is a poorly studied LINER embedded in a double ring of $\mathrm{H}$ II regions. The LINER region lies within the ring, and shows most extension in the polar direction. The velocity structure suggests that it is an outflow in the $\mathrm{NE}$ direction.

NGC 6926: A Seyfert 2 nucleus with a dynamically disturbed ENLR. This source contains an $\mathrm{H}_{2} \mathrm{O}$ megamaser (Greenhill et al. 2003). Many bright HII regions are seen in what appears to be a tidally distorted LIRG disk galaxy. A narrow-band $\mathrm{H} \alpha$ image was published by Dopita et al. (2002b). The region to the north of the nucleus may contain a low-metallicity $\mathrm{H}$ II region complex, based on its weak [N II] $\lambda 6584$ and strong [O III] $\lambda 5007$ relative to $\mathrm{H} \alpha$. We speculate that this galaxy is undergoing a minor merger.

IC 5063: A post-merger Elliptical, this galaxy containing a Seyfert 2 nucleus shows a spectacular, symmetric ENLR extending across the WiFeS field. Barrel-shaped with a bright linear core in the inner regions, it develops horns with an opening angle of $\sim 30^{\circ}$ in the outer parts. Observed with $H S T$, the inner region forms a fine knotty ionization cone in
[O III $\lambda 5007$ with an opening angle the same as in the outer regions (Schmitt et al. 2003a). A small (4 arcsec; 860pc) double-lobe plus nucleus radio structure was resolved at 8.6 GHz by Morganti et al. (1998), who also detected a very broad outflowing $\left(\sim 700 \mathrm{~km} \mathrm{~s}^{-1}\right)$ HI absorption against the strong nuclear continuum source. The broad-line nucleus was detected in polarized light by Lumsden et al. (2004). No $\mathrm{H}$ II regions are visible.

IC 1368: Seyfert 2 on old stellar continuum. The ENLR is very compact, $\lesssim 5$ arcsec, and elongated in PA $=135^{\circ}$. A few faint $\mathrm{H}$ II regions are visible in the stellar disk, which is nearly edge-on at $\mathrm{PA}=40^{\circ}$. These $\mathrm{H}$ II regions are what causes the extension in the $\mathrm{H} \alpha+\left[\mathrm{N}_{\mathrm{II}}\right]$ image of Colbert et al. (1996).

NGC 7130/IC5135: In agreement with the classification of Thuan (1984), the WiFeS nuclear spectrum shows this as mixed excitation starburst + Seyfert 2. The $[\mathrm{O}$ III $] \lambda 5007$ lines are appreciably broader than the $\mathrm{H} \beta$, and there is a strong continuum from young stars mixed with the older stellar component. Long slit spectroscopy has been obtained by Fraquelli et al. (2003). This galaxy contains ENLR $\sim 9 \times 9$ $\operatorname{arcsec}(2.7 \mathrm{kpc})$ within a bright tidally distorted barred spiral of $\mathrm{H}$ II regions, which are nicely imaged with HST (Malkan et al. 1998).

NGC 7213: This large SA galaxy contains a very bright LINER or Seyfert 1 nucleus with broad $\mathrm{H} \alpha$. An old stellar continuum is prominent in the WiFeS spectrum. Broad $\left[\mathrm{N}_{\text {II }}\right] \lambda 6584$ can be traced over $\sim 9 \times 9 \operatorname{arcsec}(0.9 \mathrm{kpc})$. A number of $\mathrm{H}$ in regions are visible toward the edge of the field; see also Evans et al. (1996). Recently Schnorr-Müller et al. (2014) presented high spatial resolution $(\sim 60 \mathrm{pc})$ integral field spectroscopy of the inner $0.8 \times 1.1 \mathrm{kpc}$ of this galaxy using the Gemini Multi-Object spectrograph, and inferred a black hole mass of $\sim 10^{8} M_{\odot}$ and a mass inflow of a few tenths of a solar mass per year.

IC 1459: A LINER with deep $\mathrm{NaD}$ absorption, and with extended diffuse emission, which can be traced to a radial distance of more than $20 \operatorname{arcsec}(>2 \mathrm{kpc})$ toward the SW in [N II $\lambda 6584$ and $\mathrm{H} \alpha$. The nucleus hosts a GPS radio source (Tingay et al. 2003), which may have features in common with NGC1052 and NGC 7213.

NGC 7469: A luminous Seyfert 1 nucleus with coronal emission and a fairly strong and extensive ENLR. A number of $\mathrm{H}$ II regions form an irregular star-forming elliptical ring approximately $20 \mathrm{arcsec}$ in diameter $(6 \mathrm{kpc})$. The ENLR is $\sim 16 \times 8 \operatorname{arcsec}(4.8 \times 2.4 \mathrm{kpc})$ in diameter.

NGC 7496: A very weak Seyfert $2+$ starburst nucleus. Like NGC 7130, the galaxy nuclear spectrum shows [O III] $\lambda 5007$ lines that are appreciably broader than $\mathrm{H} \beta$, and a strong continuum from young stars. Many HII regions, and a general outflow, are seen in $\mathrm{H} \alpha$, [N $\left.\mathrm{N}_{\mathrm{II}}\right]$, and [S II]. This extends across the field, and looks shock-excited (lines are broad and complex).

NGC 7582: This galaxy displays a star-forming disk (Cid Fernandes et al. 2001) out of which a high excitation, bright, and extended ( $>15$ arcsec, $1.3 \mathrm{kpc}$ ) ionization cone with an opening angle of $110^{\circ}$ emerges. The counter-cone also appears (although heavily reddened) on the far side of the disk, and extends across the field. The ionization cones appear to be in outflow; see also Morris et al. (1985). Many fine H II regions are embedded in the star-forming disk. An HST red continuum image is available (Malkan et al. 1998), and long slit spectroscopy has been obtained by Fraquelli et al. (2003). 
NGC 7591: The nucleus is a heavily reddened LINER with strong, old star continuum embedded in a LIRG with many $\mathrm{H}$ in regions. Zaw et al. (2009) used data from Moustakas \& Kennicutt (2006) to argue that it is simply a star-forming LIRG. However, this does not appear to be the case.

NGC 7590: A faint and compact Seyfert 2 nucleus with no apparent ENLR. Many bright HII regions are seen in the surrounding star-forming disk.

$N G C$ 7679: This Seyfert 2 galaxy with very narrow emission lines shows a very extended one-sided ionization cone emerging from a bright central disk of $\mathrm{H}$ II regions. The [O III] $\lambda 5007$ line profiles in the ENLR are complex.

NGC 7714: This starburst nucleus galaxy is interacting with the nearby galaxy NGC 7715. The nuclear spectrum shows a pure starburst with a strong blue continuum of young stars. He II emission is very clear; see also Gonzalez-Delgado et al. (1995). This galaxy has been imaged in the red continuum with HST (Malkan et al. 1998).

\section{CONCLUSIONS}

In this paper we have presented images and nuclear spectroscopy for 64 galaxies drawn from the first data release of the S7, which is available at http://miocene.anu.edu.au/priv/ S7DR1/. This data set demonstrates the utility of integral field spectroscopy of AGNs with a reasonably wide field, good spectral resolution, and a full spectral coverage in the optical. From these data we have examined the spatial relationship between the ENLR and the $\mathrm{H}$ II regions in the disks of the host galaxies. Nearly all of our Seyferts are found to have ENLR. We find that the ENLR lies inside a circumnuclear ring of star formation in $\sim 25 \%$ of the galaxies in the sample. This starforming ring is typically a few kiloparsecs across, and we may tentatively identify this feature as an Inner Lindblad Resonance. We will investigate this point on the basis of the detailed dynamics in a future paper. Where the angle of the ring to the line of sight permits, we find that the ENLR ionization cone is perpendicular to the star-forming ring, suggesting that in general, the equatorial axis of the inner accretion torus defining the ionization cones must be well aligned with the equatorial axis of the outer star-forming ring. A full examination of the dynamical structure of the ENLR, its zone of influence, the chemical abundances in the circumnuclear gas, and the constraints that can be placed on the luminosity and EUV SED of the central engine are deferred to future publications.

As far as the nuclear spectroscopy is concerned, we found interesting correlations within the class of objects that display Coronal line emission from species such as [Fe VII], [Fe X], and $[\mathrm{Fe} \mathrm{XIV]}$. We conclude that their properties are consistent with the model advocated by Mullaney et al. (2009), in which coronal lines arise in a dense gas launched from the dusty inner torus $\left(10^{17}<R / \mathrm{cm}<10^{18}\right)$ at a very high local ionization parameter $\log U \sim-0.4$, which is accelerated by radiation pressure to a terminal velocity of a few hundred $\mathrm{km} \mathrm{s}^{-1}$. For the Type 2 Seyferts, the nuclear spectra are all located in the upper region of the Seyfert sequence defined by the AGNs drawn from the SDSS galaxies on the Baldwin et al. (1981) and Veilleux \& Osterbrock (1987) diagnostic plots. We conclude that the SDSS galaxies populating the lower region of these diagrams are likely to be composite spectra caused by mixing between the Seyfert nucleus and the surrounding $\mathrm{H}$ II regions in the galaxy, and therefore the position of these objects on these diagnostic plots is likely a consequence of aperture effects in the SDSS spectra.

Note Added in Proof. Dr. Jianguo Wang of Yunnan Observatories, CAS points out that a more likely identification of the redshift of PKS 0056-572 is $z \sim 0.679$. In this, the strong emission feature at $4700 \AA$ is identified with Mg II $2800 \AA$, and the underlying emission is the Fe II $2750 \AA$ complex. The two red emission features are then $\mathrm{H} \delta$ and $\mathrm{H} \epsilon$.

M.A.D. and L.K. acknowledge the support of the Australian Research Council (ARC) through Discovery project DP130103925, M.A.D. also acknowledges financial support from King Abdulaziz University. This research began thanks to a grant from the Australian Academy of Science AustraliaIndia Visiting Fellowship (2012-2013) to M.A.D.. S.J. acknowledges support from the European Research Council through grant ERC-StG- 257720. J.S. acknowledges the European Research Council for the Advanced Grant Program Number 267399-Momentum. J.S. acknowledges support from the European Research Council through grant ERC-StG257720. This research used the NASA/IPAC Extragalactic Database (NED), which is operated by the Jet Propulsion Laboratory, California Institute of Technology, under contract with the National Aeronautics and Space Administration, the NASA Astrophysics Data System (ADS), and SAOImage DS9 (Joye \& Mandel 2003), developed by the Smithsonian Astrophysical Observatory. Last, but not least, we thank the anonymous referee for helping us to much improve this paper.

\section{REFERENCES}

Abazajian, K. N., Adelman-McCarthy, J. K., Agüeros, M. A., et al. 2009, ApJS, 182, 543

Allen, M. G., Dopita, M. A., Tsvetanov, Z. I., \& Sutherland, R. S. 1999, ApJ, 511,686

Antonucci, R. 1993, ARA\&A, 31, 473

Antonucci, R., \& Barvainis, R. 1990, ApJL, 363, L17

Baldwin, J. A., Phillips, M. M., \& Terlevich, R. 1981, PASP, 93, 5

Banfi, M., Rampazzo, R., Chincarini, G., \& Henry, R. B. C. 1993, A\&A, 280,373

Bennert, V. N., Auger, M. W., Treu, T., Woo, J.-H., \& Malkan, M. A. 2011, ApJ, 742, 107

Bicknell, G. V., Dopita, M. A., Tsvetanov, Z. I., \& Sutherland, R. S. 1998, ApJ, 495, 680

Binette, L., Magris, C. G., Stasińska, G., \& Bruzual, A. G. 1994, A\&A, 292, 13 Cappellari, M., \& Emsellem, E. 2004, PASP, 116, 138

Carollo, C. M., Stiavelli, M., de Zeeuw, P. T., \& Mack, J. 1997, AJ, 114, 2366

Cecil, G., Dopita, M. A., Groves, B., et al. 2002, ApJ, 568, 627

Childress, M. J., Vogt, F. P. A., Nielsen, J., \& Sharp, R. G. 2014, Ap\&SS, 349,617

Cid Fernandes, R., Heckman, T., Schmitt, H., González Delgado, R. M., \& Storchi-Bergmann, T. 2001, in ASP Conf. Ser. 249, The Central Kiloparsec of Starbursts and AGN: The La Palma Connection ed. J. H. Knapen, J. E. Beckman, I. Shlosman, \& T. J. Mahoney (San Francisco, CA: ASP), 536

Cid Fernandes, R., Jr., Storchi-Bergmann, T., \& Schmitt, H. R. 1998, MNRAS, 297, 579

Colbert, E. J. M., Baum, S. A., Gallimore, J. F., et al. 1996, ApJS, 105, 75

Condon, J. J. 1987, ApJS, 65, 485

Condon, J. J., \& Dressel, L. L. 1978, ApJ, 221, 456

Curran, S. J., \& Whiting, M. T. 2013, IAU Symp. 292, Molecular Gas, Dust, and Star Formation, ed. T. Wong, \& J. Ott (Cambridge: Cambridge Univ. Press), 243

Davies, R. L., Kewley, L. J., Ho, I.-T., \& Dopita, M. A. 2014a, MNRAS, 444, 3961

Davies, R. L., Rich, J. A., Kewley, L. J., \& Dopita, M. A. 2014b, MNRAS, 439, 3835

Dopita, M. A. 1997, PASA, 14, 230 
Dopita, M. A., Groves, B. A., Sutherland, R. S., Binette, L., \& Cecil, G. 2002a, ApJ, 572, 753

Dopita, M., Hart, J., McGregor, P., et al. 2007, Ap\&SS, 310, 255

Dopita, M. A., Ho, I.-T., Dressel, L. L., et al. 2015, ApJ, in press (arXiv:1501.02507)

Dopita, M., Rhee, J., Farage, C., et al. 2010, Ap\&SS, 327, 245

Dopita, M. A., Pereira, M., Kewley, L. J., \& Capaccioli, M. 2002b, ApJS, 143,47

Dopita, M. A., \& Sutherland, R. S. 2003, Astrophysics of the Diffuse Universe (Berlin: Springer)

Dopita, M. A., Scharwächter, J., Shastri, P., et al. 2014, A\&A, 566, A41

Elitzur, M. 2006, NewAR, 50, 728

Emsellem, E., Greusard, D., Combes, F., et al. 2001, A\&A, 368, 52

Evans, I. N., \& Dopita, M. A. 1987, ApJ, 319, 662

Evans, I. N., Koratkar, A. P., Storchi-Bergmann, T., et al. 1996, ApJS, 105, 93

Falcke, H., Wilson, A. S., \& Simpson, C. 1998, ApJ, 502, 199

Fathi, K., Storchi-Bergmann, T., Riffel, R. A., et al. 2006, ApJL, 641, L25

Fehmers, G. C., de Grijp, M. H. K., Miley, G. K., \& Keel, W. C. 1994, A\&AS, 108,61

Ferland, G. J., \& Netzer, H. 1983, ApJ, 264, 105

Ferrarese, L., \& Merritt, D. 2000, ApJL, 539, L9

Fischer, T. C., Crenshaw, D. M., Kraemer, S. B., Schmitt, H. R., \& Trippe, M. L. 2010, AJ, 140, 577

Fosbury, R. A. E., Mebold, U., Goss, W. M., \& Dopita, M. A. 1978, MNRAS, 183,549

Fraquelli, H. A., Storchi-Bergmann, T., \& Levenson, N. A. 2003, MNRAS, 341,449

Gebhardt, K., Bender, R., Bower, G., et al. 2000, ApJL, 539, L13

Gilli, R., Maiolino, R., Marconi, A., et al. 2000, A\&A, 355, 485

González Delgado, R. M., Cerviño, M., Martins, L. P., Leitherer, C., \& Hauschildt, P. H. 2005, MNRAS, 357, 945

Gonzalez-Delgado, R. M., Perez, E., Diaz, A. I., et al. 1995, ApJ, 439, 604

Greenhill, L. J., Kondratko, P. T., Lovell, J. E. J., et al. 2003, ApJL, 582, L11

Groves, B. A., Dopita, M. A., \& Sutherland, R. S. 2004a, ApJS, 153, 9

Groves, B. A., Dopita, M. A., \& Sutherland, R. S. 2004b, ApJS, 153, 75

Gu, Q., Melnick, J., Cid Fernandes, R., et al. 2006, MNRAS, 366, 480

Haniff, C. A., Wilson, A. S., \& Ward, M. J. 1988, ApJ, 334, 104

Heckman, T. M. 1980, A\&A, 87, 152

Ho, L. C. 1996, PASP, 108, 637

Ho, L. C., Filippenko, A. V., \& Sargent, W. L. W. 1997, ApJS, 112, 315

Kaler, J. B. 1976, ApJ, 210, 843

Kewley, L. J., Groves, B., Kauffmann, G., \& Heckman, T. 2006, MNRAS, 372,961

Kewley, L. J., Heisler, C. A., Dopita, M. A., \& Lumsden, S. 2001, ApJS, 132,37

Koski, A. T., \& Osterbrock, D. E. 1976, ApJ, 203, 10

Kriss, G. A., Hartig, G. F., Armus, L., et al. 1991, ApJL, 377, L13

Larson, D., Dunkley, J., Hishaw, G., et al. 2011, ApJS, 192, 16

Levenson, N. A., Cid Fernandes, R., Jr., Weaver, K. A., Heckman, T. M., \& Storchi-Bergmann, T. 2001, ApJ, 557, 54

Lipari, S., Tsvetanov, Z., \& Macchetto, F. 1993, ApJ, 405, 186

Lumsden, S. L., Alexander, D. M., \& Hough, J. H. 2004, MNRAS, 348, 1451

Magorrian, J., Tremaine, S., Richstone, D., et al. 1998, AJ, 115, 2285

Malizia, A., Malaguti, G., Bassani, L., et al. 2002, A\&A, 394, 801

Malkan, M. A., Gorjian, V., \& Tam, R. 1998, ApJS, 117, 25

Maoz, D., Koratkar, A., Shields, J. C., et al. 1998, AJ, 116, 55

Markwardt, C. B. 2009, in ASP Conf. Ser. 411, Astronomical Data Analysis Software and Systems 18th ed. D. A. Bohlender, D. Durand, \& P. Dowler (San Francisco, CA: ASP), 251

Matt, G., Guainazzi, M., \& Maiolino, R. 2003, MNRAS, 342, 422

Mazzuca, L. M., Knapen, J. H., Veilleux, S., \& Regan, M. W. 2008, ApJS, 174,337

Mazzalay, X., \& Rodríguez-Ardila, A. 2007, A\&A, 463, 445

McConnell, N. J., \& Ma, C.-P. 2013, ApJ, 764, 184
Moran, E. C., Barth, A. J., Kay, L. E., \& Filippenko, A. V. 2000, ApJL, $540, \mathrm{~L} 73$

Morganti, R., Killeen, N. E. B., \& Tadhunter, C. N. 1993, MNRAS, 263, 1023 Morganti, R., Oosterloo, T., \& Tsvetanov, Z. 1998, AJ, 115, 915

Morris, S., Ward, M., Whittle, M., Wilson, A. S., \& Taylor, K. 1985, MNRAS, 216, 193

Moustakas, J., \& Kennicutt, R. C., Jr. 2006, ApJS, 164, 81

Muñoz Marín, V. M., González Delgado, R. M., Schmitt, H. R., et al. 2007, AJ, 134,648

Mulchaey, J. S., Wilson, A. S., \& Tsvetanov, Z. 1996a, ApJS, 102, 309

Mulchaey, J. S., Wilson, A. S., \& Tsvetanov, Z. 1996b, ApJ, 467, 197

Mullaney, J. R., Ward, M. J., Done, C., Ferland, G. J., \& Schurch, N. 2009, MNRAS, 394, L16

Nagar, N. M., Oliva, E., Marconi, A., \& Maiolino, R. 2002, A\&A, 391, L21

Nenkova, M., Ivezić, Ž., \& Elitzur, M. 2002, ApJL, 570, L9

O’Connell, R. W., \& Dressel, L. L. 1978, Natur, 276, 374

Pereira-Santaella, M., Diamond-Stanic, A. M., Alonso-Herrero, A., \& Rieke, G. H. 2010, ApJ, 725, 2270

Phillips, M. M., Jenkins, C. R., Dopita, M. A., Sadler, E. M., \& Binette, L. 1986, AJ, 91, 1062

Pogge, R. W. 1989, ApJ, 345, 730

Preite-Martinez, A., \& Pottasch, S. R. 1983, A\&A, 126, 31

Raban, D., Heijligers, B., Röttgering, H., et al. 2008, A\&A, 484, 341

Ramos Almeida, C., Levenson, N. A., Rodríguez Espinosa, J. M., et al. 2009, ApJ, 702, 1127

Riffel, R., Rodríguez-Ardila, A., \& Pastoriza, M. G. 2006, A\&A, 457, 61

Ryder, S. D., \& Dopita, M. A. 1993, ApJS, 88, 415

Saraiva, M. F., Bica, E., Pastoriza, M. G., \& Bonatto, C. 2001, A\&A, 376, 43

Scharwächter, J., Dopita, M. A., Zuther, J., et al. 2011, AJ, 142, 43

Schmitt, H. R., Donley, J. L., Antonucci, R. R. J., Hutchings, J. B., \& Kinney, A. L. 2003a, ApJS, 148, 327

Schmitt, H. R., Donley, J. L., Antonucci, R. R. J., et al. 2003b, ApJ, 597, 768

Schmitt, H. R., \& Storchi-Bergmann, T. 1995, MNRAS, 276, 592

Schnorr-Müller, A., Storchi-Bergmann, T., Nagar, N. M., \& Ferrari, F. 2014, MNRAS, 438, 3322

Singh, R., van de Ven, G., Jahnke, K., et al. 2013, A\&A, 558, A43

Smith, J. E., Robinson, A., Alexander, D. M., et al. 2004, MNRAS, 350, 140

Storchi-Bergmann, T., Eracleous, M., Teresa Ruiz, M., et al. 1997, ApJ, 489, 87

Storchi-Bergmann, T., Nemmen da Silva, R., Eracleous, M., et al. 2003, ApJ, 598, 956

Stoy, R. H. 1933, MNRAS, 93, 588

Tadhunter, C., Morganti, R., Rose, M., Oonk, J. B. R., \& Oosterloo, T. 2014, Natur, 511, 440

Thean, A., Pedlar, A., Kukula, M. J., Baum, S. A., \& O’Dea, C. P. 2000, MNRAS, 314, 573

Thuan, T. X. 1984, ApJ, 281, 126

Tingay, S. J., Edwards, P. G., \& Tzioumis, A. K. 2003, MNRAS, 346, 327

Tran, H. D. 2003, ApJ, 583, 632

Tremaine, S., Gebhardt, K., Bender, R., et al. 2002, ApJ, 574, 740

Trippe, M. L., Crenshaw, D. M., Deo, R., \& Dietrich, M. 2008, AJ, 135, 2048

Turner, M. J. L., Reeves, J. N., Ponman, T. J., et al. 2001, A\&A, 365, L110

Veilleux, S., \& Osterbrock, D. E. 1987, ApJS, 63, 295

Veron-Cetty, M.-P., \& Veron, P. 1986, A\&AS, 66, 335

Véron-Cetty, M.-P., \& Véron, P. 2006, A\&A, 455, 773

Vogt, F. P. A., Dopita, M. A., \& Kewley, L. J. 2013, ApJ, 768, 151

Vogt, F. P. A., Dopita, M. A., Kewley, L. J., et al. 2014, ApJ, 793, 127

Ward, M., Penston, M. V., Blades, J. C., \& Turtle, A. J. 1980, MNRAS, 193,563

Wilson, A. S., Braatz, J. A., Heckman, T. M., Krolik, J. H., \& Miley, G. K. 1993, ApJL, 419, L61

Wilson, A. S., Ward, M. J., \& Haniff, C. A. 1988, ApJ, 334, 121

Yan, R., \& Blanton, M. R. 2012, ApJ, 747, 61

Zaw, I., Farrar, G. R., \& Greene, J. E. 2009, ApJ, 696, 1218

Zhang, Z. T., Liang, Y. C., \& Hammer, F. 2013, MNRAS, 430, 2605 